\title{
Serial Recall
}

\author{
Mark J. Hurlstone \\ Department of Psychology, Lancaster University, Bailrigg, Lancaster LA1 4YF, United Kingdom
}

Serial memory refers to the ability to recall a novel sequence of items or events in the correct order. In the laboratory, the dominant tool used to assess this mental faculty is the immediate serial recall (hereafter, 'serial recall') task in which participants are given a sequence of typically verbal, visual, or spatial items that they must subsequently recall in their original presentation order. Serial recall is a deceptively simple task-the apparent ease with which people accomplish it masks the wealth and complexity of findings this task has generated, and the computational theories that have been developed to account for them. In this chapter, I review benchmark findings of serial recall that have been observed across the verbal, visual, and spatial short-term memory domains, and I interpret them with reference to the core mechanisms embodied in contemporary computational theories of serial recall. This analysis identifies four mechanisms that are common to the three content domains-namely, position marking, a primacy gradient, competitive queuing, and response suppression. Additionally, evidence suggests that in verbal serial recall both the encoding and retrieval of items is sensitive to item similarity-similarity-sensitive encoding and retrieval-and that item retrieval is accompanied by output interference. By contrast, in visual and spatial serial recall there is evidence for similarity-sensitive retrieval, but the relevant empirical observations that evince similarity-sensitive encoding and output interference are yet to be studied in the visual and spatial domains. I conclude by outlining some challenges for future research.

serial recall $\mid$ serial memory $\mid$ short-term memory | verbal $\mid$ visual $\mid$ spatial $\mid$ computational models | mechanisms of serial recall

Ah, well, I don't want you to get the impression it's just a question of the number of words... um... I mean, getting them in the right order is just as important. Old Peter Hall used to say to me, 'They're all there Eddie, now we've got to get them in the right order.'

John Cleese as Sir Edwin "A Great Actor" by Monty Python

Short-term memory for serial order-the ability to store and retrieve items and events in the correct order-is the foundation for many acts of higher-order cognition. For example, serial memory supports verbal abilities such as vocabulary acquisition (Baddeley, Gathercole, \& Papagno, 1998; Page \& Norris, 2009) where the learning of a new word depends on being able to remember the phonemes that make up that word in their correct order (Baddeley et al., 1998; Gathercole \& Baddeley, 1990; Leclercq \& Majerus, 2010; Majerus, Poncelet, Greffe, \& Van der Linden, 2006). Serial memory also supports nonverbal abilities such as the acquisition of motor skills and social behaviours, which are often learned by observing and imitating sequences of actions performed by others (Agam, Bullock, \& Sekuler, 2005; Agam, Galperin, Gold, \& Sekuler, 2007; Baddeley, 2007). Furthermore, short-term memory for serial order is a core ingredient for music (Palmer \& Pfordresher, 2003), sport, and communication through speaking (Dell, Burger, \& Svec, 1997), writing (Meulenbroek, Rosenbaum, \& Thomassen et al., 1996), and typing (Logan, 2018), indicating that this cognitive ability is essential to human cultural behaviour more generally.

In the laboratory, the dominant task used to assess serial memory is the serial recall or memory span task (Box 1). In this task, participants are given a sequence of verbal, visual, or spatial items that they must subsequently reproduce in forward serial order, either immediately or following a brief delay. This task is one of the oldest and best known in experimental psychology. In the years since it was introduced it has generated a wealth of robust empirical findings that have served as targets for both verbal and computational theorising. The main challenge for any adequate account of performance on this task is to provide a solution to the problem of serial order (Lashley, 1951)-how people store and recall a novel sequence of items in the correct order. However, serial recall is deceptively simple-the apparent ease with which people perform it masks a complex set of underlying mechanisms-and introspection and intuition are poor guides to theorising about how this task is performed.

A popular verbal account of how people accomplish this serial recall task is based on a working memory model (Baddeley \& Hitch, 1974) comprising a phonological loop-dedicated to the retention of verbal sequences-and a visuospatial sketchpaddedicated to the retention of visuospatial sequences. The latter system contains two separate components: a "visual cache"dedicated to the retention of visual sequences-and an "inner scribe"-dedicated to the retention of spatial sequences (Logie, 1995). These subsystems are augmented by a central executive that coordinates their activities, and an episodic buffer that provides an interface with long-term memory (Baddeley, 2000). Although this model has been hugely influential and offers a qualitative account of the effects of a number of key variables on serial recall performance, a widely acknowledged criticism (e.g., Burgess \& Hitch, 1992; Hurlstone \& Hitch, 2015; Hurlstone, Hitch, \& Baddeley, 2014 ) is that it fails to offer a mechanistic account of how the serial recall task is actually accomplished within the two short-term memory sub-systems, leaving the fundamental problem of serial order unresolved.

${ }^{1}$ Department of Psychology, Lancaster University, Bailrigg, Lancaster LA1 4YF, United Kingdom

To whom correspondence should be addressed. E-mail: mark.hurlstonelancaster.ac.uk 


\section{Box 1 Serial recall}

In the serial recall task, participants are given a sequence of items-typically conveyed at a rate of 0.5 to $1 \mathrm{~s}$ per item-that they must subsequently reproduce in forward serial order. In verbal serial recall (a), the items are typically letters, digits, or words presented visually or aurally. Serial recall is accomplished by speaking, writing, or typing the items in turn. In a variant of this task, known as serial order reconstruction (Healy, 1982; Healy, Fendrich, Cunningham, \& Till, 1987), in the reproduction phase the items are simultaneously represented in a jumbled visual array and participants must mouse click on the items in their original presentation order. In visual serial recall (b), the items are typically unfamiliar faces (e.g., Smyth, Hay, Hitch, \& Horton, 2005) or novel black and white matrix patterns (e.g., Avons \& Mason, 1999). Due to the absence of a natural response mode for these stimuli, sequence reproduction necessarily requires the use of serial order reconstruction. In spatial serial recall, the items are either visual-spatial (c; Jones, Farrand, Stuart, \& Morris, 1995) or auditory-spatial (d; Parmentier \& Jones, 2000) locations, or visual-spatial movements of a disc (e; Agam et al., 2005). In c, serial recall is accomplished by mouse clicking on the locations, which are concealed from view, whereas in serial order reconstruction the locations are visible in the reproduction phase. In d, serial recall is accomplished by manually pointing to each sound-specified location, whereas in serial order reconstruction the locations are represented by icons on a computer display and participants mouse click on the icons in the presentation order of the locations they represent. In e, serial recall is achieved by retracing the movements of the disc with a graphics stylus.

Given that serial recall and serial order reconstruction produce comparable results, in this chapter, I will use the term "serial recall" to refer collectively to results obtained using both sequence reproduction protocols.

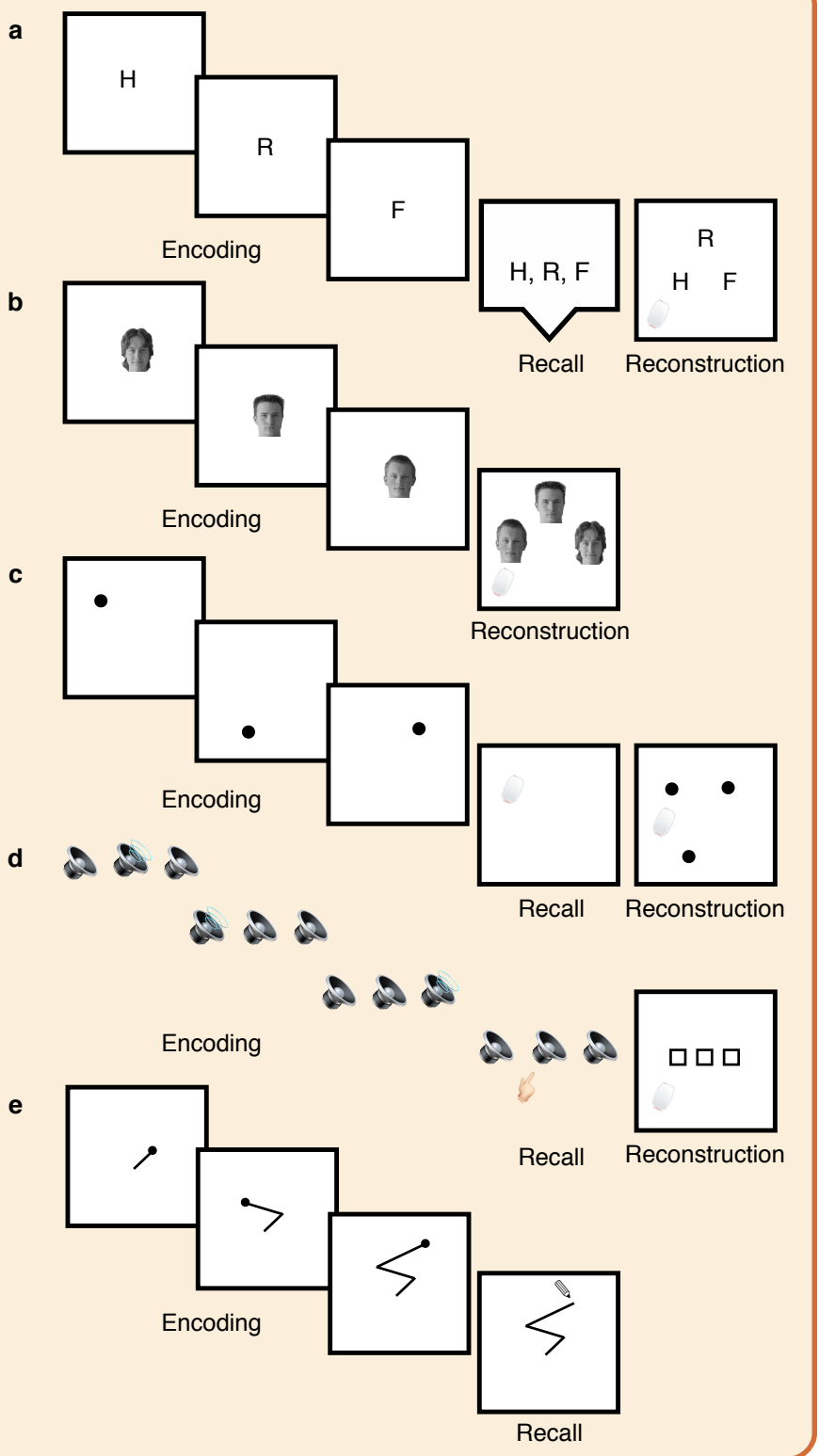

To date, no computational theories of serial recall in the nonverbal visual and spatial domains have been developed. This is because due to experimental convenience-and the perceived centrality of serial order processing to language - the lions share of research using the serial recall task has employed verbal materials as stimuli. Indeed, studies of serial recall using visual and spatial materials only got under way as contemporary computational theories of verbal serial recall were being developed in the mid 1990s. However, since that time, there has been a steady rise in the number of empirical investigations of visual and spatial serial recall. What these studies have shown is that most of the benchmark effects of verbal serial recall generalise to the serial recall of visual and spatial materials. This suggests that mechanisms of serial recall instantiated in theories of verbal serial memory are also candidate mechanisms for theories of visual and spatial serial memory.

In this chapter, I review benchmark effects of serial recall observed with verbal, visual, and spatial materials, and I interpret 
Table 1 | Benchmark effects of serial recall and the content domains (verbal, visual, spatial) in which they have been demonstrated.

\begin{tabular}{|c|c|c|c|}
\hline \multirow[b]{2}{*}{ Benchmark } & \multicolumn{3}{|c|}{ Content domain } \\
\hline & Verbal & Visual & Spatial \\
\hline \multicolumn{4}{|l|}{ 1. Serial position curve } \\
\hline 1.1. Primacy & $\checkmark$ & $\checkmark$ & $\checkmark$ \\
\hline 1.2. Recency & $\checkmark$ & $\checkmark$ & $\checkmark$ \\
\hline 2. Sequence length effect & $\checkmark$ & $\checkmark$ & $\checkmark$ \\
\hline \multicolumn{4}{|l|}{ 3. Error patterns } \\
\hline 3.1. Transposition gradients & $\checkmark$ & $\checkmark$ & $\checkmark$ \\
\hline 3.2. Transposition latencies & $\checkmark$ & $\checkmark$ & $\checkmark$ \\
\hline 3.3. Fill-in: infill ratio & $\checkmark$ & $\checkmark$ & $\checkmark$ \\
\hline 3.4. Intrusions & $\checkmark$ & $\checkmark$ & $\checkmark$ \\
\hline 3.5. Protrusions & $\checkmark$ & $?$ & $?$ \\
\hline 3.6. Omissions & $\checkmark$ & $?$ & $\checkmark$ \\
\hline 3.7. Repetitions & $\checkmark$ & $\checkmark$ & $\checkmark$ \\
\hline 3.8. More order than item errors & $\checkmark$ & $\checkmark$ & $\checkmark$ \\
\hline \multicolumn{4}{|l|}{ 4. Temporal grouping effects } \\
\hline 4.1. Grouping advantage & $\checkmark$ & $\checkmark$ & $\checkmark$ \\
\hline 4.2. Within group primacy \& recency & $\checkmark$ & $\checkmark$ & $\checkmark$ \\
\hline 4.3. Interpositions & $\checkmark$ & $x$ & $x$ \\
\hline 4.4. Product rule & $\checkmark$ & $?$ & $?$ \\
\hline \multicolumn{4}{|l|}{ 5. Item similarity effects } \\
\hline 5.1. Pure sequences & $\checkmark$ & $\checkmark$ & $\checkmark$ \\
\hline 5.2. Mixed sequences (standard) & $\checkmark$ & $\checkmark$ & $?$ \\
\hline 5.3. Mixed sequences (revised) & $\checkmark$ & $?$ & $?$ \\
\hline \multicolumn{4}{|l|}{ 6. Ranschburg effect } \\
\hline 6.1. Repetition inhibition & $\checkmark$ & $?$ & $?$ \\
\hline 6.2. Repetition facilitation & $\checkmark$ & $?$ & $?$ \\
\hline 7. Temporal isolation (non) effect & $\checkmark$ & $\checkmark$ & $\checkmark$ \\
\hline
\end{tabular}

these effects in relation to the core mechanisms embodied in computational theories of serial recall. The goal of this review is to identify the mechanisms of serial recall across the verbal, visual, and spatial domains, and, in so doing, establish whether these mechanisms are the same across the three domains. The review is an abridged and updated version of an earlier review that addressed the same questions (Hurlstone et al., 2014).

\section{Benchmark effects of serial recall}

Given its long history in the cognitive psychology literature, the serial recall task has generated a wide range of empirical results which cannot be readily summarised. However, not all results are created equal in terms of their theoretical importance. Results that lack generalisability and the theoretical leverage to differentiate between competing theories should receive less priority than results that possess these attributes (Oberauer et al., 2018). Accordingly, in this chapter I focus on seven benchmark findings, and sub-findings, that are well replicated, have been shown (in most instances) to generalise across content domains (verbal, visual, spatial), and have demonstrably been shown to have the capacity to adjudicate between rival mechanisms and theories of serial recall*.

"In this chapter, I omitt two of the benchmarks previously included in my earlier review (Hurlstone et al. 2014)—backward serial recall and the Hebb repetition effect. Backward recall is omitted because it is arguably the least diagnostic of the benchmarks considered by Hurlstone et al. (2014), given the multifarious ways it can be accomplished. The Hebb repetition effect-the long term learning of a sequence that is surreptitiously repeated-is omitted due to my focus here on the "serial recall" task specifically - rather than "memory for serial order" more generally (as was the focus of my earlier review) - which refers to the recall of novel sequences of items, rather than the long-term learning of the same sequence.

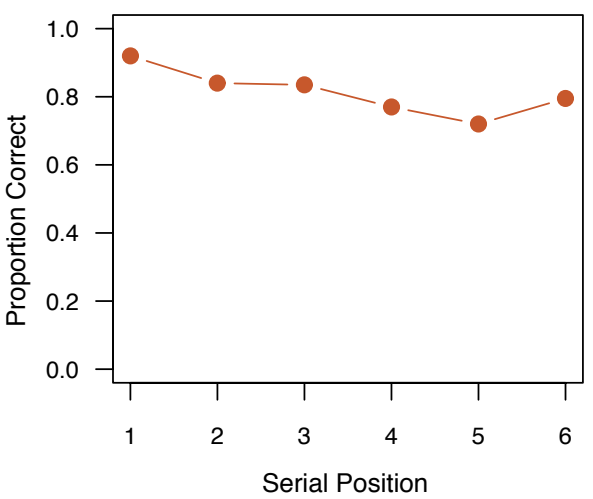

Figure 1 Serial position curve for verbal serial recall of 6-item sequences of phonologically dissimilar consonants. Data from Henson, Norris, Page, and Baddeley (1996, Experiment 1).

1.1. Serial position curve. The serial position curve plots recall accuracy as a function of the serial positions of items. The serial position curve associated with verbal serial recall is characterised by two canonical effects that have been replicated across countless studies (Figure 1): First, there is a sharp monotonic decrease in recall accuracy extending from the first position onwards known as the primacy effect. Second, there is a small upturn in performance for the final serial position known as the recency effect.

Accuracy serial position curves exhibiting effects of primacy and recency are not confined to verbal serial recall. They have also been observed for visual serial recall of matrix patterns (Avons, 1998; Avons \& Mason, 1999), and unfamiliar faces (Smyth et al., 2005; Ward, Avons, \& Melling, 2005), and spatial serial recall of auditory-spatial locations (Groeger, Banks, \& Simpson, 2007; Parmentier \& Jones, 2000; Tremblay, Guérard, Parmentier, Nicholls, \& Jones, 2006), visual-spatial locations (Avons, 2007; Farrand, Parmentier, \& Jones, 2001; Guérard \& Tremblay, 2008; Jones et al., 1995; Smyth \& Scholey, 1996; Tremblay et al., 2006), and visual-spatial movements (Agam et al., 2005; Agam et al., 2007; Agam, Huang, \& Sekuler, 2010).

1.2. Sequence length effect. Verbal serial recall accuracy decreases with increasing sequence length (Anderson, Bothell, Lebiere, \& Matessa, 1998; Crannell \& Parrish, 1957; Maybery, Parmentier, \& Jones, 2002). This sequence length effect has also been documented for visual serial recall of matrix patterns (Avons, 1998) and unfamiliar faces (Hurlstone \& Hitch, 2018; Smyth et al., 2005; Ward et al., 2005), and spatial serial recall of visual-spatial locations (Jones et al., 1995; Smyth, 1996; Smyth, Pearson, \& Pendleton, 1989; Smyth \& Scholey, 1994, 1996) and visual-spatial movements (Agam et al., 2005, Agam et al., 2007).

1.3. Error patterns. Errors in serial recall can be transposition errors or item errors. A transposition occurs when an item from the study sequence is recalled in the wrong position. When plotted over serial position, transpositions in verbal serial recall obey an inverted $U$ shaped trend, with most occurring at medial sequence positions (Henson, 1996; Henson, Norris, Page, \& Baddeley, 1996). The incidence of transpositions in visual serial recall of matrix patterns (Avons \& Mason, 1999) and spatial serial recall of visualspatial locations (Guérard \& Tremblay, 2008) has also been shown to conform to this inverted $U$ shaped trend.

Transpositions can be classified according to their displace- 


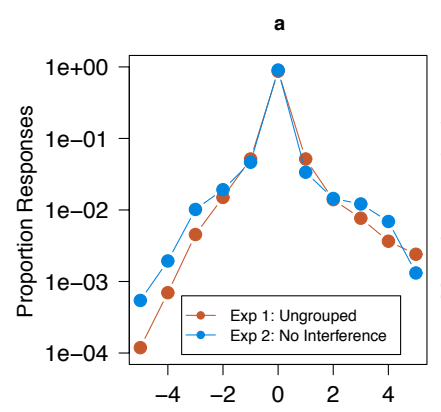

Transposition Displacement

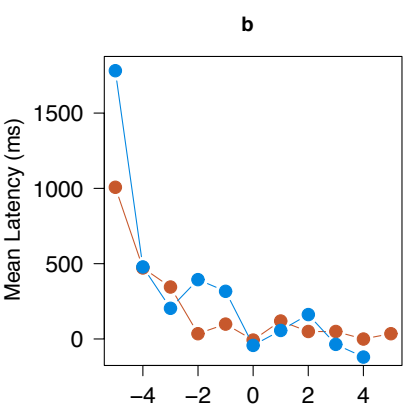

Transposition Displacement
Figure 2 | Response probabilities and recall latencies of transposition errors for verbal serial recall of 6-item sequences of digits or consonants. Transposition errors are measured in terms of transposition displacement-the numeric difference between an item's input and output position. A displacement value of zero refers to a correct response. A negative displacement refers to an anticipation error (e.g., a displacement of -2 corresponds to an item recalled two positions ahead of its correct position) whereas a positive displacement refers to a postponement error (e.g., a displacement of +4 corresponds to an item recalled four positions after its correct position). Panels show transposition error gradients (a), which plot the proportion of recalled items as a function of transposition displacement, and latency-displacement functions (b), which plot the mean recall latency of recalled items as a function of transposition displacement. Note that the negative recall latencies at some displacement values in the latter panel are a consequence of filtering out the confounding effect of output position on recall latencies (by subtracting the mean recall latency at each output position from the individual recall latencies at those positions for each participant). To explain, recall latencies in serial recall become gradually quicker as output position increases (see e.g., Farrell \& Lewandowsky, 2004), which can artificially lengthen the recall latencies of anticipations (which predominantly occur at early output positions) and artificially shorten the recall latencies of postponements (which predominantly occur at late output positions). Data from Farrell and Lewandowsky (2004, Experiments 1 \& 2).

ment, which refers to the numerical difference between an item's presentation and recall positions. Transpositions with negative displacement values are known as anticipation errors and correspond to items recalled ahead of their correct positions. Transpositions with positive displacement values are known as postponement errors and correspond to items recalled after their correct positions. Items recalled in their correct positions are represented by a displacement value of zero. Transpositions are typically measured in terms of transposition gradients which plot the probability of transpositions as a function of displacement. Typical transposition gradients for verbal serial recall are shown in Figure 2a from which it can be seen that the probability of an error decreases as the absolute displacement value increases-thus, when an item is recalled in the wrong position it will tend to be close to its correct position. This tendency for transpositions to cluster around their correct recall positions is known as the locality constraint (Henson, 1996) The locality constraint is not confined to verbal serial recall. Transposition gradients for visual serial recall of matrix patterns (Avons \& Mason, 1999) and unfamiliar faces (Smyth et al., 2005), and spatial serial recall of visual-spatial locations (Parmentier, Andrés, Elford, \& Jones, 2006; Smyth \& Scholey, 1996), auditory-spatial locations (Groeger et al., 2007; Parmentier \& Jones, 2000; Parmentier, King, \& Dennis, 2006), and visual-spatial movements (Agam et al., 2005) have also been shown to display this fundamental property.

The transposition gradient for verbal serial recall is accompanied by a systematic pattern of recall latencies. Farrell and Lewandowsky (2004) have shown that when the latency of transpositions is plotted as a function of displacement-a so-called latency-displacement function-anticipations are slower than postponements, as illustrated in Figure 2b. Additionally, it can be seen that transposition displacement has different effects on the recall latencies for anticipations and postponements: Latencies for anticipations increase as an approximately linear function of displacement, whereas latencies for postponements are generally invariant with respect to displacement. This latency-by-displacement pattern has since been replicated for visual serial recall of unfamiliar faces (Hurlstone \& Hitch, 2018) and spatial serial recall of visual-spatial locations (Hurlstone \& Hitch, 2015).

A final feature of transpositions has also turned out to be important for identifying computational principles underpinning memory for serial order. This is that transposition errors in verbal serial recall are characterised by a particular pattern of sequential dependency. Specifically, if an item $i$ is recalled a position too soon, recall of item $i-1$ is more likely at the next output position than item $i+1$. To explain, given the sequence $A B C$, if $B$ is recalled at the first output position then a fill-in error, reflected by the recall of $A$ at the next output position, is more likely than an infill error, reflected by the recall of $\mathrm{C}$. Available data on these errors suggests that fill-in errors outweigh infill errors by a ratio of approximately 2:1 (Farrell, Hurlstone, \& Lewandowsky, 2013; Henson, 1996; Osth \& Dennis, 2015a; Surprenant, Kelley, Farley, \& Neath, 2005). This fill-in tendency extends to spatial serial recall of visual-spatial locations (Guérard \& Tremblay, 2008) and visual serial recall of unfamiliar faces (Hurlstone \& Hitch, 2018), and it obeys the approximate 2:1 ratio observed with verbal serial recall.

There are boundary conditions to the fill-in tendency. In a reanalysis of four published serial recall experiments, Solway, Murdock, and Kahana (2012) observed the opposite pattern of sequential dependency, with infill errors outweighing fill-in errors. There are several procedural differences between the experiments just reviewed on the fill-in tendency and those examined by Solway et al. (2012). Their experiments employed longer sequences (between 10 and 19 items), and required participants to remember words rather than letters or digits. In addition, their experiments did not require precise placement of items - the requirement instead was that any items that were recalled should be recalled in their relative order of presentation, such that participants were free to skip any number of items without penalty. By contrast, in typical serial recall experiments participants are required to report items in their precise location and also to record omissions (see next) explicitly if these are allowed. Accordingly, sequence length, the nature of the recall instructions given to participants, and the type of stimuli used (consonants and digits vs. words) are important determinants of the resultant pattern of sequential error dependency. However, under typical serial recall protocols, fill-in errors dominate over infill errors.

Item errors can be divided into intrusion, omission, and repetition errors. An intrusion occurs when an item is recalled that was not part of the study sequence. In verbal serial recall, intrusions often involve the recall of an item on trial $n$ that occurred in the same within-sequence position on trial $n-1$ (Conrad, 1960; Henson, 1999; Osth \& Dennis, 2015b). These position-preserving intrusions are known as protrusions (Henson, 1996). An omission occurs when an item is not recalled, whilst a repetition occurs when an item is recalled on more than a single occasion despite being presented only once in the study sequence. Repetitions are rare in verbal serial recall accounting for approximately $2 \%$ (Henson, 1996) to $5 \%$ (Vousden \& Brown, 1998) of all responses. Repetitions are even rarer in visual and spatial serial recall (Hurlstone \& Hitch, $2015,2018)$, accounting for less than $1 \%$ of all responses.

In verbal serial recall, item errors are less common than transposition errors, accounting for around $20 \%$ of total errors (Aaronson, 
a

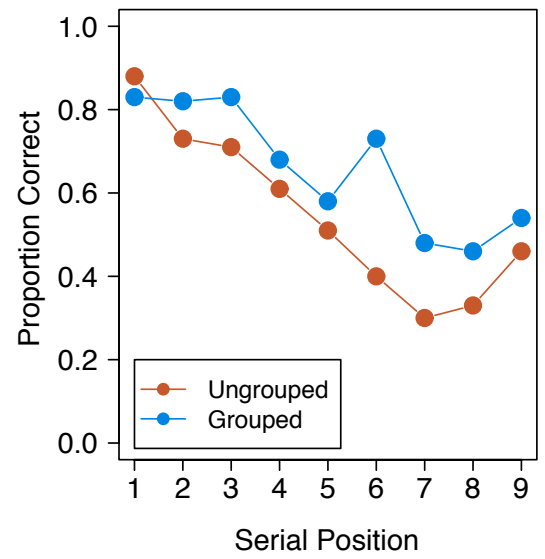

b

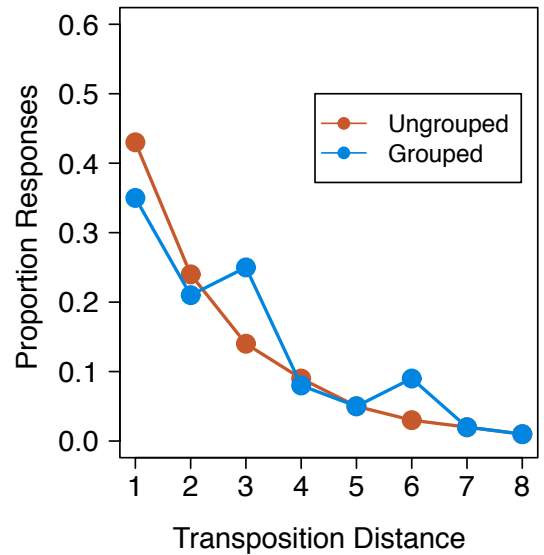

c

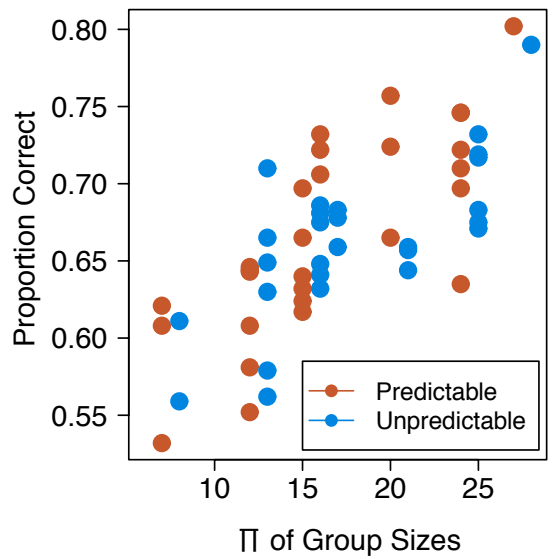

Figure 3 Temporal grouping effects for verbal serial recall of 9-item sequences of digits: accuracy serial position curves (a), and transposition error gradients (b) for sequences grouped in a 3-3-3 pattern, and scatterplot of the relationship between the product of the group sizes of 27 different grouping patterns and recall accuracy under predictable and unpredictable study conditions (c). Data from Hurlstone (2018) (a-b) and Hartley et al. (2016) (c).

1968), and their incidence has been shown to increase across serial positions (Henson, 1996). Guérard and Tremblay (2008) have shown that item errors are also less frequent than transpositions for spatial serial recall of visual-spatial locations and that the incidence of intrusions and omissions (the only item errors reported in their study) increases across serial positions. Avons and Mason (1999) observed a similar pattern for visual serial recall of matrix patterns using a serial reconstruction method that permitted the recording of transpositions and intrusions.

1.4. Temporal grouping effects. Differentiating a sequence into sub-groups by inserting extended temporal pauses after every few items-known as temporal grouping-has been shown to exert a number of systematic effects on verbal serial recall. First, compared to an ungrouped baseline, grouping enhances recall accuracy (Frankish, 1985, 1989; Henson, 1996; 1999; Hitch, Burgess, Towse, \& Culpin, 1996; Maybery et al., 2002; Ng \& Maybery, 2005; Ryan, 1969a, 1969b). Second, grouping produces effects of primacy and recency within each sub-group (Figure 3a), giving the accuracy serial position curve for grouped sequences a scalloped appearance (Frankish, 1985, 1989; Hitch et al., 1996). Third, grouping modifies the pattern of errors by reducing the number of transpositions overall, and between groups in particular. However, one type of between group transposition actually increases in grouped sequences: These interpositions are transpositions between groups that preserve their positions within groups (Henson, 1996, 1999a; Ng \& Maybery, 2002, 2005; Ryan, 1969a). For example, if a 9-item sequence is organised into three groups of three, interpositions are indicated by an increase in the probability of \pm 3 and \pm 6 transpositions (Figure $3 b$ ).

With the exception of the increase in interpositions in grouped sequences, the remaining effects of temporal grouping just reviewed have been reproduced using visual serial recall of unfamiliar faces (Hurlstone \& Hitch, 2018), and spatial serial recall of visual-spatial locations (Hurlstone, 2018; Hurlstone \& Hitch, 2018; Parmentier, Andrés et al., 2006) and auditory-spatial locations (Parmentier, Maybery, \& Jones, 2004).

Another feature of temporal grouping hitherto only examined with verbal serial recall was initially documented by Ryan (1969b).
She presented participants with spoken sequences of nine digits that were temporally grouped into three groups of varying sizes (e.g., 2-6-1, 4-4-1, 2-3-4, and 3-3-3) yielding 27 different grouping patterns conveyed in a random order. Ryan (1969b) observed considerable heterogeneity in the effectiveness of the different grouping patterns, and noted that the efficacy of temporal grouping appears to be a function of the regularity of the grouping. Recently, Hartley et al. (2016) replicated and extended Ryan's results by showing that even when participants have foreknowledge of the forthcoming grouping pattern it has no beneficial effect on serial recall. That is, comparing recall accuracy on the 27 different grouping patterns under conditions where the grouping pattern on the forthcoming trial was known versus unknown revealed no reliable differences in performance. Furthermore, Hartley et al. (2016) identified a simple empirical yardstick to explain the substantial variation in performance across different groupings. Known as the product rule, this rule states that as the product of the group sizes for a given grouping pattern increases, so too does recall performance (Figure $3 \mathrm{c}$ ). The product rule provides a metric for the degree of regularity of the grouping pattern-small products are associated with irregular groupings, where one group is much larger than the others, whereas the largest product and highest level of recall corresponds to equal groups of three. The product rule suggests the efficacy of temporal grouping is a function of the regularity of the grouping, as Ryan (1969b) originally suggested.

1.5. Item similarity effects. A classic and robust finding in the verbal short-term memory literature is that sequences of phonologically similar sounding items (e.g., $B D G P T V$ ) are recalled less accurately than sequences of phonologically dissimilar sounding items (e.g., F K $R X Y$; Baddeley, 1966, 1968; Conrad, 1964; Wickelgren, 1965a, 1965b). This phonological similarity effect (Baddeley, 1986) is also observed when sequences are constructed by alternating phonologically dissimilar and similar items (e.g., FBKGRT). Such mixed sequences engender a sawtoothed accuracy serial position curve characterised by peaks corresponding to the recall of dissimilar items and troughs corresponding to the recall of similar items (Baddeley, 1968; Farrell, 2006; Farrell \& Lewandowsky, 2003; Henson et al., 1996; Lewandowsky 
\& Farrell, 2008b). Representative data for this mixed-sequence phonological similarity effect are shown in Figure 4a.

Initial studies of this mixed-sequence phonological similarity effect showed that dissimilar items in mixed sequences are recalled with the same level of accuracy as items in corresponding positions in pure dissimilar sequences (Baddeley, 1968; Henson et al., 1996) — a result dubbed the dissimilar immunity finding (Farrell, 2006). However, recent studies have cast doubt on the validity of this finding (Farrell, 2006; Farrell \& Lewandowsky, 2003; Lewandowsky \& Farrell, 2008b). Farrell and Lewandowsky (2003) showed that the absence of a difference in the recall accuracy of dissimilar items on pure and mixed sequences is a consequence of a failure to equate the stimulus ensemble sizes for the two sequence-types. In early studies (Baddeley, 1968; Henson et al. 1996), the stimulus ensembles for mixed sequences contained double the number of items used in the pure dissimilar and similar sequence stimulus ensembles. Farrell and Lewandowsky showed that the effect of this imbalance is to increase the number of omissions and intrusions in mixed sequences. As can be seen in Figure $4 \mathrm{~b}$, when the greater incidence of these errors in mixed sequences was abolished by equating the stimulus ensemble sizes for the two sequence types, Farrell and Lewandowsky found that dissimilar items on mixed sequences were recalled with greater accuracy than their counterparts on pure dissimilar sequences. This socalled mixed-sequence advantage is of considerable empirical generality, having been witnessed using: (a) immediate serial recall (Farrell, 2006; Farrell \& Lewandowsky, 2003; Lewandowsky \& Farrell, 2008b), (b) delayed serial recall (Farrell, 2006), (c) blocked (Farrell, 2006; Farrell \& Lewandowsky, 2003) and random presentation (Lewandowsky \& Farrell, 2008b) of pure and mixed sequences, and (d) mixed sequences containing equal numbers of dissimilar and similar items or a single dissimilar item appended to a sequence of otherwise similar items (Farrell, 2006; Farrell \& Lewandowsky, 2003; Lewandowsky \& Farrell, 2008b).

Item similarity effects have also been documented in visual serial recall where they manifest as visual similarity effects. Avons and Mason (1999) found that sequences of purely visually similar matrix patterns were recalled less accurately than sequences of purely visually dissimilar matrix patterns, whilst Smyth et al. (2005) found that sequences of purely visually similar unfamiliar faces were recalled less accurately than sequences of purely visually dissimilar unfamiliar faces. Moreover, using visually similar and dissimilar Japanese Kanji characters, Logie et al. (Logie, Saito, Morita, Varma, \& Norris, 2016) demonstrated both pure sequence and mixed sequence visual similarity effects reminiscent of those observed in verbal serial recall. Logie et al. (2016) did not control for guessing strategies by equating the stimulus ensembles for pure and mixed sequences and failed to observe a mixedsequence advantage. It therefore remains to be seen whether a mixed-sequence advantage would be observed in visual serial recall when guessing strategies are controlled. Jalbert, Saint-Aubin, and Tremblay (2008) have shown an item similarity effect in spatial serial recall. They found that sequences of spatial locations presented in the same color hue were recalled less accurately than sequences of locations presented in different color hues.

1.6. Ranschburg effects. The Ranschburg effect (named after its discoverer: Paul Ranschburg)—also known as the phenomenon of repetition inhibition-occurs when two conditions are compared in the serial recall of verbal sequences: In the repetition condition, the sequences presented for recall contain two occurrences of the same item separated by a number of intervening items, whilst in the
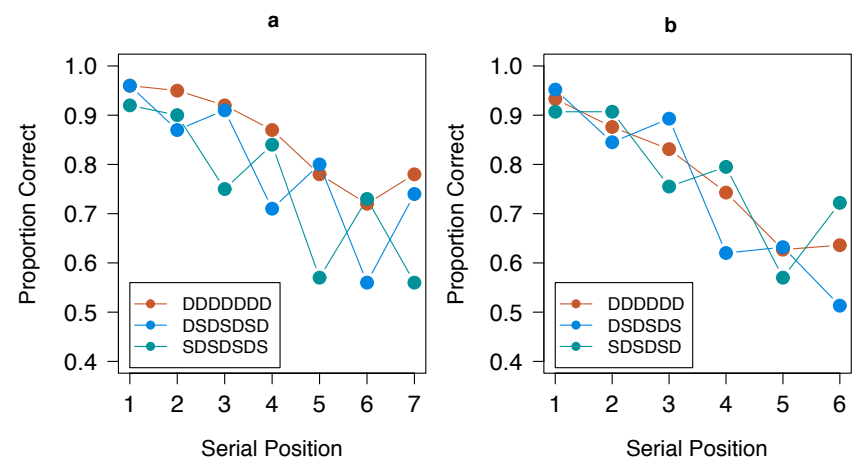

Figure 4 | Accuracy serial position curves for verbal serial recall of alternating sequences of phonologically similar (S) and dissimilar (D) consonants-with similar consonants at odd positions (SDS...) or even positions (DSD...) - and purely phonologically dissimilar sequences of consonants (DDD...). Data show the standard mixed sequence phonological similarity effect illustrating the dissimilar immunity finding (a) and the mixed-sequence advantage that results when guessing strategies are controlled (b). Data from Henson et al. (1996, Experiment 2) (a) and Lewandowsky and Farrell (2008b; Experiment 2) (b)

control condition, the sequences always contain unique items. The typical finding is that recall of the second occurrence of a repeated item is impaired relative to items in corresponding positions in the control condition (Crowder, 1968; Duncan \& Lewandowsky, 2005; Henson, 1998b; Jahnke, 1969; Kahana \& Jacobs, 2000; Vousden \& Brown, 1998). A violation of this general pattern occurs when the two occurrences of a repeated item are presented within close proximity-adjacent or separated by a single position. Under these conditions, recall of both occurrences of a repeated item is often enhanced relative to items in corresponding positions in the control condition-a phenomenon known as repetition facilitation (Crowder, 1968). The Ranschburg effect has not yet been investigated in visual or spatial serial recall.

1.7. Temporal isolation (non) effect. Owing to a debate in the short-term memory literature regarding whether the psychological dimension of time plays a key role in the representation of serial order, several studies have examined whether serial recall exhibits so-called temporal isolation effects. Items are said to be temporally isolated if they are surrounded by relatively long temporal gaps compared to other items in the study sequence. To test for possible temporal isolation effects, Neath and Crowder $(1990,1996)$ used increasing (e.g., A . B .. C ... D .... E ..... F) or decreasing (A ..... B .... C ... D .. E . F) temporal presentation schedules in a verbal serial recall task. They found that items near the beginning of the sequence-which are more temporally isolated in the decreasing than the increasing condition-were recalled with greater accuracy in the decreasing condition, whereas items toward the end of the sequence-which are more temporally isolated in the increasing than the decreasing condition-were recalled more accurately in the increasing condition.

At first blush, these results provide confirmatory evidence that temporal isolation influences verbal serial recall. However, the problem with using increasing and decreasing temporal presentation schedules is that participants can predict the location of the extended temporal gaps in advance based on the known sequence structure. Thus, rather than reflecting a pure effect of time, it is possible that participants simply use their foreknowledge of the location of the extended temporal gaps to strategically allocate more time to rehearsing the temporally isolated items. It follows 
that a proper test of the temporal isolation hypothesis requires the use of random and unpredictable temporal presentation schedules. More recent experiments that meet this constraint have shown no effect of temporal isolation in verbal serial recall (Farrell, 2008; Lewandowsky \& Brown, 2005; Lewandowsky, Brown, Wright, \& Nimmo, 2006; Nimmo \& Lewandowsky, 2005, 2006; Parmentier, King, \& Dennis, 2006), visual serial recall (Peteranderl \& Oberauer, 2018), or spatial serial recall (Parmentier et al., 2006).

\section{Mechanisms of serial recall}

The wealth and complexity of contemporary computational theories of serial recall means that a thorough treatment of each is beyond the purview of this chapter. Moreover, a focus on the detailed properties of specific models can obscure important commonalities that exist between them. Fortunately, due to a certain degree of co-evolution in their development, there has been some theoretical convergence amongst theories, and several mechanisms of serial recall have now been identified which are commonly employed. Accordingly, I classify theories according to the core mechanisms on which they rely to produce their behaviour (cf. Hurlstone et al., 2014; Lewandowsky \& Farrell, 2008a). I begin by considering mechanisms for the representation of serial order, which include (a) associative chaining, (b) position marking, and (c) a primacy gradient. I then consider the additional mechanisms that augment or support these seriating mechanisms, which include (d) competitive queuing, (e) response suppression, (f) output interference, and (g) similarity-sensitive encoding and retrieval.

\subsection{Seriating mechanisms.}

2.1.1. Associative chaining. Associative chaining is the oldest solution to the problem of serial order (Ebbinghaus, 1885/1964; Kahana, 2012) and the mechanism of serial recall in several computational theories (Lewandowsky \& Murdock, 1989; Murdock, 1993, 1995; Solway et al., 2012). In chaining models, serial order is encoded by forming associations between study items. Serial recall is accomplished by traversing these associations, which serve as the retrieval cues for sequence production. For example, given the study sequence $A, B, C$, retrieval of $A$ will cue retrieval of $B$, which will then cue retrieval of $C$. Chaining models can be divided into two classes: simple chaining and compound chaining. In simple chaining models (Figure 5a), such as the original Theory of Distributed Associative Memory (TODAM) model (Lewandowsky \& Murdock, 1989), only forward associations between adjacent items are used to represent serial order. By contrast, in compound chaining models (Figure 5b; Solway et al., 2012), which includes later instantiations of TODAM (Murdock, 1993, 1995), serial order is represented by forward and backward associations between both adjacent and non-adjacent items, the strength of which decreases gradually as a function of the distance between items, with backward associations being weaker than forward associations (as indicated by the dashed lines in Figure 5b). An additional assumption required in both simple and compound chaining models is that the first item in the sequence must be associated with a start of sequence marker that can be used as a cue to kickstart the chaining process at recall.

2.1.2. Position marking. Position marking is an approach to representing serial order where each item in a sequence is associated with the current state of an internal context signal that changes gradually during sequence presentation. At recall, the context signal is reset and re-evolves along its original path, with sequence items being activated according to the degree of similarity between the current state of the context signal and the state to which each item was associated.

Models that use position marking to represent serial order can be classified as event-based, time-based, or a hybrid of the two. In event-based models, the context signal changes only when a new event (e.g., a new item) is experienced. In one class of event-based models, the context signal encodes absolute withinsequence position. Models falling into this class include C-SOB (Farrell, 2006; Lewandowsky \& Farrell, 2008a) and the original Burgess and Hitch (1992) model. For example, in the latter model, items are associated with a context signal implemented as a vector of inactive nodes containing a dynamic window of active nodes. The context vector changes gradually with the presentation of each item by sliding the attentional window from left to right by a constant one node per item. In another class of event-based models, the context signal encodes relative within-sequence position. For example, in the Start-End Model (SEM; Henson, 1998a; see also Houghton, 1990) items are linked to the varying states of a context signal comprising two elements-a start marker that is strongest for the first position and decreases exponentially in strength across positions, and an end marker that is weakest for the first position and increases exponentially in strength across positions. Such a context signal represents approximate position relative to the start and end of the sequence

In time-based models, the context signal changes as a function of absolute time. Models in this class include more recent instantiations of the Burgess and Hitch $(1999,2006)$ model in which the same moving window context signal changes with time rather than events, and the Oscillator-Based Associative Recall (OSCAR) model (Brown et al., 2000). In the OSCAR model, items are linked with the different states of a time-varying context signal driven by sets of temporal oscillators operating at different frequencies. At recall the context signal is reset to its initial state before being replayed, with list items being re-activated through their original associations with the timing signal. A major advantage of this model is that it specifies an explicit and neurally plausible mechanism for generating the context signal. A similar, but more abstract, temporal coding scheme is utilised by the SIMPLE model (Brown et al., 2007).

Notwithstanding its specification of an explicit mechanism for deriving the context signal, one limitation of OSCAR is that the timing signal is based on free-running oscillators that are not influenced by bottom-up properties of the input sequence, but rather their own interconnectivity. The bottom-up entrainment of oscillators is not implemented in this model, as Brown et al. (2000) were careful to note. Recently, Hartley et al. (2016) have presented a model of auditory-verbal serial recall known as BUMP (standing for Bottom-Up Multi-scale oscillator Population) that addresses this limitation. In the BUMP model, instead of using free-running oscillators, the timing signal is based on the activity of a population of oscillators acting as temporal filters. The oscillators are sensitive to amplitude modulations in the envelope of incoming speech, with each oscillator possessing an intrinsic tuning-a tendency for its activity to oscillate at a specific rate and phase. The frequency tunings of the oscillators are chosen to span the range of presentation rates encountered in the serial recall task. In BUMP, when a sequence is presented-modelled as an input signal based on amplitude modulations associated with presentation of each itemthe rhythm and timing of items determines which oscillators in the 


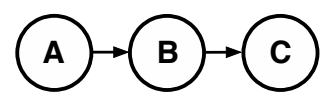

C

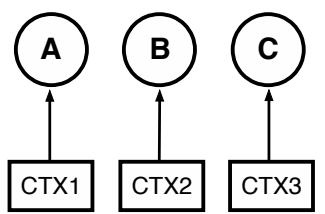

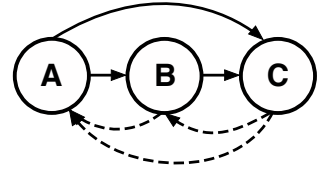

d

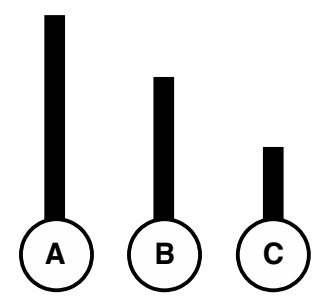

Figure 5 | Mechanisms for the representation of serial order: simple chaining (a), compound chaining (b), position marking (c), and primacy gradient (d).

BUMP population become entrained to the bottom-up input. This bottom-up on the fly composition of the context signal based on the information in the physical stimulus, as it unfolds in real time, solves two fundamental problems-how to anticipate the start and end of a sequence, and choosing the appropriate rate of change of the context signal for a given task. The BUMP model is a hybrid model-it is time-based in that the context signal changes smoothly over time, but it is event-based in that these changes are driven by events.

2.1.3. Primacy gradient. A simpler scheme for representing serial order is in terms of a primacy gradient of activation strength, whereby the first item is activated strongest and the activations of subsequent items decrease monotonically across positions. When serial order is represented by a primacy gradient complemented by response suppression (see section 2.2.2.), serial recall is accomplished via an iterative process of selecting the most active item for recall before suppressing its activation so the next strongest item can be selected. This is the functional mechanism for ordered recall in the models of Grossberg (1978a, 1978b), the primacy model (Page \& Norris, 1998), the Serial-Order-in-a-Box (SOB) model (Farrell \& Lewandowsky, 2002), and the LIST PARSE (Laminar Integrated Storage of Temporal Patterns for Associative Retrieval, Sequencing and Execution) model (Grossberg \& Pearson, 2008). Some models that use position marking to represent serial order also incorporate a primacy gradient (e.g., Brown et al., 2000; Burgess \& Hitch, 1999; Farrell, 2006; Henson, 1998a; Lewandowsky \& Farrell, 2008a). For example, in OSCAR (Brown et al., 2000) and C-SOB, (Farrell, 2006; Lewandowsky \& Farrell, 2008a), the primacy gradient is implemented as an exponential decrease in the strength of the associations between items and their position markers.

Although most models of serial recall incorporate a primacy gradient, they differ in terms of the mechanism they adopt to explain its genesis. For example, in the SOB model (Farrell \& Lewandowsky, 2002) and its more recent extension, C-SOB (Lewandowsky \& Farrell, 2008a), the primacy gradient is a consequence of an endogenous encoding process, known as similarity-sensitive encoding. This process determines the encoding strength of each study item by calculating its novelty with respect to existing information in memory. Items which are dissimilar—and thus novel—compared to existing information in memory are encoded strongly, whereas

items which are similar-and thus less novel-compared to existing information in memory are encoded less strongly. Crucially, because each new study item will bear some resemblance to existing information in memory this means that each item will be encoded with less strength than its predecessor, thereby generating a primacy gradient. Other models rely on past-item buffering (Grossberg \& Pearson, 2008), decaying inhibition (Burgess \& Hitch, 1999), or one or two weighting parameters (Brown et al., 2000; Henson, 1998a; Page \& Norris, 1998) to generate the primacy gradient. However, as we will see later in section 3.7. encoding conditions exist in which SOB and C-SOB predict a non-monotonic rather than a monotonic primacy gradient.

\subsection{Mechanisms of selection, suppression, interference, and similarity-sensitivity.}

2.2.1. Competitive queuing. Most contemporary computational theories of serial recall use a two-stage parallel sequence planning and control mechanism known as competitive queuing (Figure 6; Houghton, 1990). In the first stage, target items are (re)activated in parallel by the ordering mechanism driving serial recall. There are two types of activating mechanisms-static and dynamic (Glasspool, 2005). In competitive queuing models using static mechanisms (Farrell \& Lewandowsky, 2002; Page \& Norris, 1998), the activating mechanism generates a single activation gradient (viz., a primacy gradient) over the target items that is then held static during recall. These activated representations are copied to the second stage, wherein items compete for selection with one another through self excitation and mutual inhibition between items based on their activation levels. The item with the strongest activation level is selected for recall, after which its corresponding representation in the first stage is inhibited, either partially or fully (viz., response suppression; see section 2.2.2.). This competition is re-run until all items have been selected for output in the second stage and their corresponding representations in the first stage have been inhibited. Competitive queuing models employing dynamic activating mechanisms (Botvinick \& Plaut, 2006; Brown et al., 2000; Burgess \& Hitch, 1992, 1999, 2006; Grossberg \& Pearson, 2008; Hartley et al., 2016; Henson, 1998a; Lewandowsky \& Farrell, 2008a) operate in a similar way, except that in the first stage the activating mechanism generates a dynamic activation gradient that varies with the passage of time or recall events (viz., position marking).

The major features of competitive queuing are that: (a) items are (re)activated in parallel at retrieval, (b) item selection occurs on the basis of a parallel search for a maximum through winnertakes-all dynamics, and (c) sequential inhibition of the activation of retrieved items eliminates them from the competitive queue to prevent erroneous repetitions of the same item, and facilitate sequence generation.

2.2.2. Response suppression. Response suppression refers to the inhibition or removal of items from memory following recall and is an assumption incorporated in almost all theories of serial recall (e.g., Brown et al., 2000; Burgess \& Hitch, 1999; Farrell \& Lewandowsky, 2002; Grossberg \& Pearson, 2008; Hartley et al., 2016; Henson, 1998a; Lewandowsky \& Farrell, 2008a; Lewandowsky \& Murdock, 1989; Page \& Norris, 1998). In models that rely on a primacy gradient to represent serial order, the incorporation of response suppression is crucial for sequencing, since it serves to prevent perseveration on the same response. It is a less crucial ingredient in models that rely on position marking to represent serial 


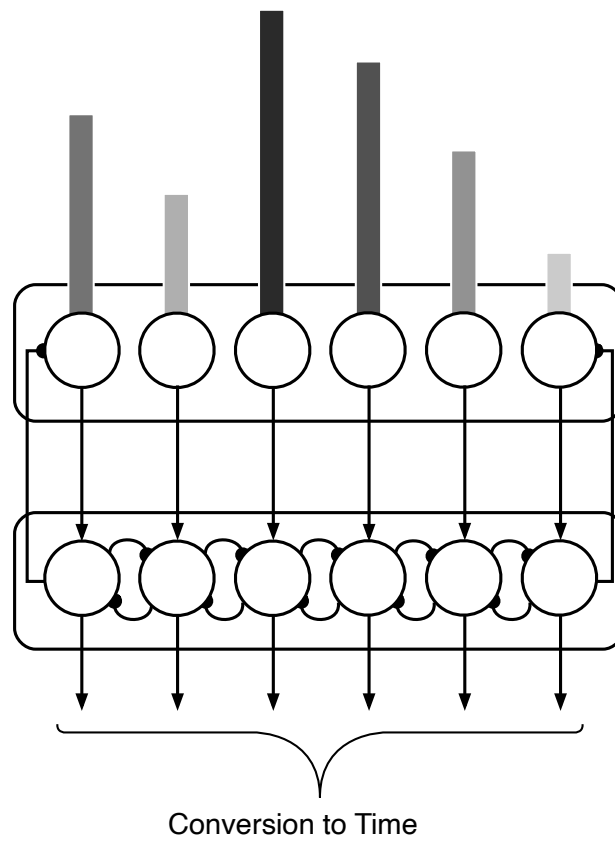

Activation Layer (Stage 1)

Selection Layer (Stage 2)

Figure 6 | Schematic of a two-stage competitive-queuing sequence planning and control mechanism comprising an activation layer (stage 1; upper field of nodes) and a selection layer (stage 2; lower field of nodes). Lines terminating with arrows represent excitatory connections, whereas lines terminating with semicircles represent inhibitory connections. Note that each node in the lower selection layer has an inhibitory connection to every other node in the same layer, but for simplicity only adjacentneighbor inhibitory connections are shown. Similarly, each node in the selection layer has an inhibitory connection to its corresponding node in the activation layer-the pathway through which response suppression (see section 2.2.2.) is implementedbut to avoid visual clutter only feedback connections for the leftmost and rightmost nodes are illustrated. The columns in the activation layer represent the activation levels of the nodes representing items in the to-be-recalled sequence. These activations may be generated by a primacy gradient established during serial order encoding which produces a single activation gradient that is held static during recall (a so-called static activating mechanism in competitive queuing terminology), or the activation gradient may change dynamically during recall in response to the reinstatement of different position markers to which items were associated during serial order encoding (a so-called dynamic activating mechanism in competitive queuing terminology). The activations in this layer are projected to the selection layer where items compete for selection via mutual inhibition between items and self excitation. The strongest item is selected for recall after which its corresponding representation in the activation layer is suppressed (viz., response suppression) via the inhibitory pathway from the selection layer to the activation layer. This process repeats until all items have been chosen for output in the selection layer, and their representations in the activation layer have been suppressed.

order because during recall the dynamically re-evolving context signal continuously modifies the activation level of items, thereby relieving the suppression mechanism of the burden for sequencing. Nevertheless, even models that represent serial order via position marking must incorporate response suppression to minimise the occurrence of erroneous repetitions, which occur infrequently in serial recall (Henson, 1996; Hurlstone \& Hitch, 2015, 2018; Vousden \& Brown, 1998).

2.2.3. Output interference. An additional mechanism implemented in the TODAM (Lewandowsky \& Murdock, 1989), OSCAR (Brown et al., 2000), C-SOB (Lewandowsky \& Farrell, 2008a), and SIMPLE (Brown et al., 2007) models of serial recall is that of output interference. This refers to the notion that the recall of an item interferes with the representations or accessibility of items that are yet to be retrieved. This output interference manifests irrespective of whether or not serial order is represented by associative chaining between items, position marking, or a primacy gradient, and regardless of whether or not items are suppressed once they have been retrieved. Since the effects of output interference accumulate as sequence production unfolds, the representations of items in the middle and towards the end of a sequence will be most impaired by its action. Accordingly, output interference is one mechanism by which the primacy effect in serial recall might materialise. Output interference can also help explain the sequence length effect, as the build-up of proactive interference rises gradually with increasing sequence length.

2.2.4. Similarity-sensitive encoding and retrieval. Several theories additionally incorporate assumptions about the nature and locus of item similarity effects (Botvinick \& Plaut, 2006; Brown et al., 2007; Burgess \& Hitch, 1999; Farrell, 2006; Henson, 1998a; Lewandowsky \& Farrell, 2008a; Page \& Norris, 1998). These theories can be distinguished according to whether they explain similarity effects solely in terms of the stage of retrieving item information, or whether similarity also affects initial encoding.

According to retrieval-based accounts of similarity effects (Burgess \& Hitch, 1999; Henson, 1998a; Page \& Norris, 1998), ordered recall proceeds in (at least) two competitive stages. In the first, order-based competition stage, items are activated by the ordering mechanism driving recall and the strongest item is chosen. In the second, similarity-based competition stage, an item chosen from the first stage will undergo a further competition in which it is vulnerable to confusion with other items remaining to be recalled based upon its degree of similarity to those items. For example, in the primacy model (Page \& Norris, 1998) items are activated according to a primacy gradient in the first stage and the item with the strongest activation is selected through competitive queuing. The recalled item is then passed on to a second stage wherein its activation is set equal to 1 . Items which are similar to the recalled item are activated by an amount equal to the value of a parameter reflecting their degree of similarity, whereas items which are dissimilar to the recalled item have an activation equal to 0 . The effect of this is to increase the likelihood that a similar item recalled from the first stage will be confused with another similar item in the second stage, thus accommodating the poorer ordered recall of sequences of similar items.

Other theories (Botvinick \& Plaut, 2006; Brown et al., 2007; Farrell, 2006; Lewandowsky \& Farrell, 2008a), by contrast, posit that the effects of similarity do not occur solely during retrieval, but also occur during the encoding of serial order. For example, the CSOB model (Farrell, 2006; Lewandowsky \& Farrell, 2008a) has an initial serial ordering stage that involves context-item associations and a second retrieval stage in which a "noisy" item representation recovered from the first stage is converted into a recallable item. This latter "deblurring" stage uses long-term knowledge in order to reconstruct degraded representations of items retrieved from the first stage. C-SOB predicts an effect of similarity on serial order encoding by virtue of the similarity-sensitive encoding mechanism that underlies the generation of its primacy gradient. Recall from earlier that similarity-sensitive encoding determines the encoding strength of each successive study item by computing its similarity to the current contents of memory. Items that are dissimilar and novel with respect to existing information in memory are encoded strongly, whereas items that are similar are encoded less strongly. A natural consequence of this similarity-sensitive encoding process is that items in similar sequences will be encoded with less strength than items in dissimilar sequences, rendering the primacy 
Table 2 | Phenomena of serial recall, the content domains in which they have been documented, and the serial recall mechanisms they are directly attributable to based on the accompanying analysis. $\mathrm{PG}=$ primacy gradient; $\mathrm{OI}=$ output interference; $\mathrm{RS}=$ response suppression; $\mathrm{PM}=$ position marking; $\mathrm{EB}+\mathrm{TB}=$ event-based + time-based; SS-E = similarity-sensitive encoding; SS-R = similarity-sensitive retrieval.

\begin{tabular}{|c|c|c|c|c|c|}
\hline \multirow[b]{2}{*}{ Phenomena } & \multirow[b]{2}{*}{ Representative study } & \multicolumn{3}{|c|}{ Content domain } & \multirow[b]{2}{*}{ Inferred mechanisms } \\
\hline & & Verbal & Visual & Spatial & \\
\hline \multicolumn{6}{|l|}{ Serial position curve } \\
\hline Primacy (input position) & Oberauer (2003) & $\checkmark$ & ? & ? & $P G$ \\
\hline Primacy (output position) & Oberauer (2003) & $\checkmark$ & $?$ & $?$ & Ol \\
\hline Conditional recency & Farrell \& Lewandowsky (2012) & $\checkmark$ & $?$ & $?$ & RS \\
\hline \multicolumn{6}{|l|}{ Error patterns } \\
\hline Transposition latencies & Farrell \& Lewandowsky (2004) & $\checkmark$ & $\checkmark$ & $\checkmark$ & $P G+P M+R S$ \\
\hline Fill-in: infill ratio & Farrell et al. (2013) & $\checkmark$ & $\checkmark$ & $\checkmark$ & $P G+R S$ \\
\hline Protrusions & Henson (1999) & $\checkmark$ & $?$ & $?$ & PM \\
\hline Repetitions & Duncan \& Lewandowsky (2005) & $\checkmark$ & $\checkmark$ & $\checkmark$ & RS \\
\hline \multicolumn{6}{|l|}{ Temporal grouping effects } \\
\hline Grouping advantage & Hitch et al. (1996) & $\checkmark$ & $\checkmark$ & $\checkmark$ & PM \\
\hline Within group primacy \& recency & Hitch et al. (1996) & $\checkmark$ & $\checkmark$ & $\checkmark$ & PM \\
\hline Interpositions & Ryan (1969a) & $\checkmark$ & $x$ & $x$ & $\mathrm{PM}(\mathrm{EB}+\mathrm{TB}) ?$ \\
\hline Product rule & Hartley et al. (2016) & $\checkmark$ & $?$ & $?$ & $\mathrm{PM}(\mathrm{EB}+\mathrm{TB}) ?$ \\
\hline \multicolumn{6}{|l|}{ Item similarity effects } \\
\hline Pure sequences & Henson et al. (1996) & $\checkmark$ & $\checkmark$ & $\checkmark$ & SS-R \\
\hline Mixed sequences (standard) & Henson et al. (1996) & $\checkmark$ & $\checkmark$ & ? & SS-R \\
\hline Mixed sequences (revised) & Farrell \& Lewandowsky (2003) & $\checkmark$ & $?$ & $?$ & $S S-E+S S-R+P G$ \\
\hline Ranschburg effect & Henson (1998b) & $\checkmark$ & $?$ & $?$ & $\mathrm{RS}$ \\
\hline Temporal isolation (non) effect & Lewandowsky et al. (2006) & $\checkmark$ & $\checkmark$ & $\checkmark$ & $\mathrm{PM}(\mathrm{EB}+\mathrm{TB}) ?$ \\
\hline
\end{tabular}

gradient for similar sequences shallower than that for dissimilar sequences. In addition to its effect on encoding, similarity affects recall by reducing the accuracy of the deblurring process used to disambiguate retrieved items. Both the effects of similarity at encoding and during retrieval in C-SOB render errors more likely in similar sequences than in dissimilar sequences.

\section{Mechanism evaluation and selection}

I now review the evidence for the operation-or lack thereof-of the seven mechanisms of serial recall reviewed in section 2 , in an attempt to identify the theoretical constructs that must be implemented in any adequate account of serial memory in the three content domains. This task is rendered difficult by the fact thatbecause the different models and mechanisms of serial recall were developed to explain a common set of findings-they can account for many of the benchmark results reviewed in section 1 equally well, thus preventing theoretical adjudication between them on the basis of these results. Fortunately, however, several of the benchmarks listed in Table 1-as well as some new data that I introduce next-do provide direct support for specific mechanisms of serial recall. To foreshadow the main conclusions of this section, Table 3 summarises those benchmarks, the content domains in which they have been documented, and the mechanisms of serial recall they are directly attributable to based on the foregoing analysis.

3.1. Associative chaining. Models that rely on associative chaining to represent serial order face a number of challenges. Although the chaining mechanism produces a primacy effect, it produces no recency effect. The primacy effect arises because successful recall of item $i$ depends on the correct recall of item $i-1$, which in turn depends upon the correct recall of item $i-2$. This dependency means that the chaining mechanism predicts recall performance will decrease monotonically across serial positions, with perfor- mance being worst at the final position. Furthermore, although the chaining mechanism generates a primacy effect, without ancillary mechanisms the extent of primacy produced will tend to be weaker than observed empirically. To accurately model the primacy effect, Lewandowsky and Murdock (1989) had to augment TODAM with two mechanisms-a primacy gradient in the encoding strength of each successive association, and output interference during recall. Similarly, to explain the recency effect, two mechanisms were once again implemented-retroactive interference during the encoding of item and associative information, and response suppression (see section 3.5. for further explanation). Whilst independent empirical evidence can be adduced for each of these mechanisms, the combination of all four to explain two of the most basic serial recall benchmarks is hardly parsimonious.

Simple chaining models also encounter difficulties explaining the locality constraint on transposition errors-since a simple chaining mechanism only activates forthcoming items, it cannot readily explain how an earlier item can take the place of a later one as an error. Compound chaining models, by contrast, can capture the pattern of transpositions by virtue of their use of bidirectional and graded associations between items (Murdock, 1995; Solway et al., 2012). Omission and intrusion errors are also problematic for strong versions of the chaining hypothesis, since in both cases the cue for the next to-be-recalled item will have been lost, meaning serial recall must terminate before the end of the sequence is reached (although see Lewandowsky \& Murdock, 1989 for a solution to this problem in the case of omissions). This prediction is at variance with the behavioural data, since participants frequently do recover from such errors.

These challenges notwithstanding, there are more serious objections to chaining. First, chaining accounts have difficulties explaining the pattern of findings associated with the recall of sequences containing repeated items. For example, given the sequence $A B A C$, chaining accounts predict that recall of $B$ and 
$C$ will be compromised, because they share the same retrieval cue. However, the Ranschburg effect shows that it is the recall of the second occurrence of the repeat that is impaired, not the items following the repeats. Second, a related problem occurs when participants are given sequences containing alternating phonologically similar and dissimilar items, such as the sequence $B K P R$. Chaining accounts predict that recall of the dissimilar items $K$ and $R$ should be impaired, because they possess similar (confusable) retrieval cues. However, as we have seen, this prediction is contrary to the data (Baddeley, 1968; Henson et al., 1996) which shows that dissimilar items on mixed sequences are recalled as effectively as items in corresponding positions on pure dissimilar sequences, if not more so (see e.g., Farrell, 2006; Farrell \& Lewandowsky, 2003; Lewandowsky \& Farrell, 2008b). Third, chaining accounts predict more infill than fill-in errors, because an item recalled too soon will subsequently cue the item that followed it in the input sequence more strongly than any other by virtue of its direct associative link with that item. This prediction is consistent with the empirical pattern observed under the atypical serial recall protocols reported by Solway et al. (2012) but it is at variance with the empirical pattern observed under typical serial recall protocols (Farrell et al., 2013; Guérard \& Tremblay, 2008; Henson et al., 1996; Hurlstone \& Hitch, 2018; Page \& Norris, 1998; Surprenant et al., 2005).

3.2. Position marking. Models that use position marking to represent serial order can account for effects of primacy, recency, and sequence length, the locality constraint, and the increase in omissions and intrusions across output positions (Brown et al., 2000; Burgess \& Hitch, 1999; Henson, 1998a; Lewandowsky \& Farrell, 2008a). Primacy and recency effects are partly, if not wholly, determined by "edge effects"- there are less opportunities for items near the beginning and end of a sequence to move around, compared to items at medial positions. In some models (e.g., Brown et al., 2007; Henson, 1998a), an additional contributing factor is the greater distinctiveness of the context signal at terminal positions. Sequence length effects arise because the greater the number of items in the target sequence, the greater the probability of an error being committed. In some models (e.g., Henson, 1998a), an additional factor contributing to the sequence length effect is that the resolution of the positional codes for longer sequences is weaker than for shorter sequences. The locality constraint arises because of the local self-similarity of the context signal, rendering adjacent-neighbour transpositions most common. Omission errors are accommodated by incorporating an output threshold that the strongest item must exceed in order to be recalled, whilst intrusion errors are modelled by weakly activating extra-sequence items to allow them to enter into the competition process. The increase in omissions and intrusions over output positions is attributable to the primacy gradient that virtually all positional models incorporate (Brown et al., 2000; Burgess \& Hitch, 1999; Henson, 1998a; Lewandowsky \& Farrell, 2008a). The encoding strength of items decreases across serial positions rendering items towards the end of the sequence more likely to fall beneath the output threshold or encounter strong competition from extra-sequence items (by contrast, the increase in repetitions across output positions arises because as output position increases there are more opportunities to produce these errors).

There are two sources of direct evidence for position marking, the first being temporal grouping effects (e.g., Frankish, 1985, 1989; Hitch et al., 1996; Maybery et al., 2002; Ryan, 1969a, 1969b). To accommodate grouping effects, models assume a more complex hierarchically organised context signal with one component of the signal representing the positions of items or groups within the sequence, and with the second component of the signal representing the positions of items within groups (Brown et al., 2007; Brown et al., 2000; Burgess \& Hitch, 1999; Hartley et al., 2016; Henson, 1998a; Lewandowsky \& Farrell, 2008a). The combination of these two signals has been shown to be necessary and sufficient to accommodate the major effects of grouping on recall accuracy and errors (Brown et al., 2000; Hartley et al., 2016; Henson, 1998a; Lewandowsky \& Farrell, 2008a).

The second piece of direct evidence for position marking is positional errors in serial recall-namely interpositions in temporally grouped sequences (Henson, 1996, 1999a; Ng \& Maybery, 2002, 2005; Ryan, 1969a) and protrusions in ungrouped sequences (Conrad, 1960; Henson, 1999; Osth \& Dennis, 2015b). Positional models of serial recall predict interpositions in grouped sequences, because items in the same positions in different groups will be associated with the same states of the component of the context signal that tracks within-group position, rendering them vulnerable to confusion. Protrusions can be accommodated by assuming that as well as representing the position of items in a sequence, the context signal also represents the position of items within a sequence of sequences (Burgess \& Hitch, 1999; Henson, 1998a). Such errors manifest because items occupying the same sequence position on different trials will be associated with the same states of the context signal that represents within-sequence position, rendering them vulnerable to confusion. Theories that represent serial order using a primacy gradient cannot accommodate protrusions, because the simple gradient-based representation of order does not provide any direct coding of positional information.

One debate revolves around whether the data support eventbased or time-based accounts of position marking. Two sources of experimental evidence have been used to adjudicate between these two accounts. The first source of evidence comes from experiments by $\mathrm{Ng}$ and Maybery $(2002,2005)$ who compared eventbased and time-based accounts on the basis of their predictions regarding the locus of interpositions in temporally grouped sequences. Event-based accounts predict that interpositions should preserve their ordinal within-group position-items that occur in the same ordinal position in different groups should exchange position with one another. By contrast, time-based accounts predict that interpositions should maintain their temporal within-group position-items that occur at the same time in different groups should exchange position with one another. Across four experiments that varied the presentation rates of items in different temporal groups, $\mathrm{Ng}$ and Maybery $(2002,2005)$ observed a pattern of between-group confusions consistent with event-based accounts, and at variance with time-based accounts.

The second source of evidence concerns the temporal isolation (non) effect, which is at variance with time-based account of serial recall such as OSCAR (Brown et al., 2000), SIMPLE (Brown et al, 2007), and the Burgess and Hitch model (Burgess \& Hitch, 1999, 2006). These theories predict that the states of the context signal to which temporally isolated items are associated should be more distinctive than the states to which temporally non-isolated items are associated. Accordingly, temporal isolation should benefit short-term serial recall which-as we have seen-is at variance with the empirical data (Farrell, 2008; Lewandowsky \& Brown, 2005; Lewandowsky et al., 2006; Nimmo \& Lewandowsky, 2005, 2006; Parmentier et al., 2006; Peteranderl \& Oberauer, 2018).

Although these results are incompatible with purely time-based accounts, they are less problematic for the BUMP model of Hartley 
et al. (2016), since it possesses the characteristics of both eventand time-based accounts. Its event-based characteristics arise from its sensitivity to local changes in amplitude of the speech signal, whereas its time-based characteristics arise from the continuous change in the context signal. Moreover, BUMP is able to account for a benchmark result that is beyond the purview of extant event-based or time-based models-namely, the product rule. Hartley et al. (2016) report detailed simulations showing that BUMP provides an excellent quantitative account of both their own and Ryan's (1969a) data involving different patterns of temporal grouping. It is BUMP's on the fly construction of the context signal and sensitivity to the rhythm and timing of the bottom-up input that enables it to reproduce this benchmark.

Given that BUMP is the only model that can explain the results of $\mathrm{Ng}$ and Maybery $(2002,2005)$, the temporal isolation (non) effect, and the product rule, whilst also specifying an explicit neurally plausible process implementation of the context signal, I tentatively interpret these benchmarks as supporting a model that uses a hybrid (event-based + time-based) representation of position (Table 2).

3.3. Primacy gradient. Models that rely on a primacy gradient to represent serial order can also explain many of the key benchmarks of serial recall. When complemented by response suppression, these models can account for the extensive primacy and restricted recency of the accuracy serial position curve, the sequence length effect, and the locality constraint (Farrell \& Lewandowsky, 2002, 2004; Page \& Norris, 1998). The primacy effect materialises because the activations of items near the beginning of the sequence are more distinctive, meaning these items encounter less competition during recall than items towards the end of the sequence. By contrast, the recency effect manifests because as successive items are recalled and suppressed, the number of response competitors is gradually reduced. Thus, as the end of the sequence approaches, only one or two unsuppressed items will compete for recall in final sequence positions. The sequence length effect occurs because increases in the length of the target sequence will necessarily increase the probability of committing at least one error during recall, whilst the locality constraint arises because the disparity in activation between items is smallest for those at neighbouring ordinal positions. Primacy gradient models can also account for the increase in omissions and intrusions across output positions (Farrell \& Lewandowsky, 2002; Page \& Norris, 1998), the mechanisms for explaining these errors being the same as those described in section 3.2.

However, there are two findings that support a direct role for a primacy gradient. First, a primacy gradient, complemented by response suppression, is necessary to accommodate the finding that fill-in errors are more frequent than infill errors (Farrell et al., 2013; Guérard and Tremblay, 2008; Henson, 1996; Hurlstone \& Hitch, 2018; Osth \& Dennis, 2015a; Surprenant et al., 2005). Primacy gradient models predict this empirical outcome, because if an item $i$ is recalled a position too soon and then suppressed, item $i-1$ will be a stronger competitor at the next recall position than item $i+1$, because the former item, by virtue of being presented earlier in the sequence, will have been encoded more strongly on the primacy gradient. Although some theories that use position marking to represent serial order can also accommodate this result (Burgess \& Hitch, 1999; Henson, 1998a), they do so either by incorporating a primacy gradient as one component of the context signal responsible for coding positional information (Henson, 1998 a) or by incorporating a primacy gradient in conjunction with

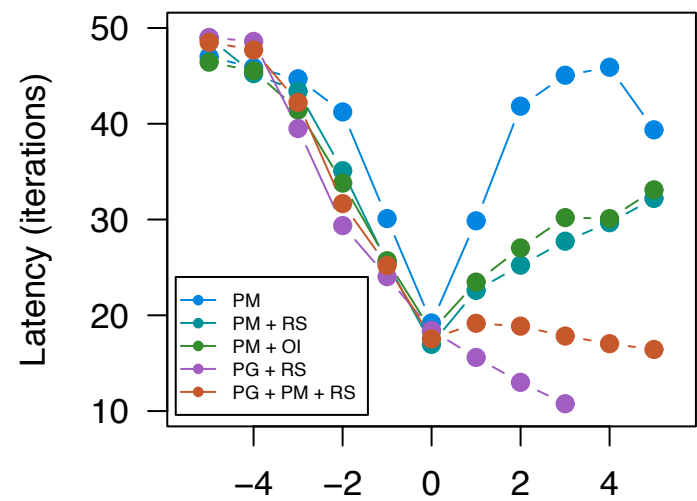

Transposition Displacement

Figure 7 | Predicted latency-displacement functions for five models and mechanisms for the representation of serial order. The functions plot the mean recall latency of recalled items as a function of transposition displacement-the numeric difference between an item's presentation and recall position. Recall that a displacement value of zero refers to a correct response, a negative displacement refers to an anticipation error, whereas a positive displacement refers to a postponement error. When serial order is represented via position marking (PM) alone, the function relating recall latency to displacement is symmetric, whereas the addition of output interference $(\mathrm{PM}+\mathrm{OI})$ or response suppression $(\mathrm{PM}+\mathrm{RS})$ renders the function partially asymmetric. In sharp contrast, when serial order is represented by a primacy gradient in conjunction with response suppression $(P G+R S)$, the function relating recall latency to displacement is monotonically negative. Finally, the addition of position marking to the combination of a primacy gradient and response suppression $(P G+P M+R S)$ renders the slope of the function for postponements flat, without removing the overall negative latency-displacement relationship. $P M=$ position marking; $P G=$ primacy gradient; $\mathrm{RS}$ = response suppression; $\mathrm{OI}=$ output interference. Predictions from Hurlstone and Hitch (2018).

position marking (Burgess \& Hitch, 1999). Farrell et al. (2013) have shown that a model combining a primacy gradient, position marking, and response suppression can explain both the tendency for fill-in errors to dominate infill errors under standard serial recall protocols, and the tendency for infill errors to dominate fill-in errors with longer sequences studied under serial learning conditions in which participants are able to skip over items (cf. Solway et al., 2012). Thus, although the different patterns that can be observed might imply a dissociation, Farrell et al. (2013) have shown that they can be explained by a common model and relate to whether or not spontaneously formed groups of items can be skipped over during recall.

Second, a primacy gradient is necessary to accommodate the pattern of transposition latencies witnessed across content domains (Farrell \& Lewandowsky, 2004; Hurlstone \& Hitch, 2015, 2018). Figure 7 shows the latency-displacement functions predicted by five models of serial order examined by Farrell and Lewandowsky (Farrell \& Lewandowsky, 2004; Lewandowsky \& Farrell, 2008a). It can be seen by inspection of this figure that when serial order is represented on the basis of position marking alone (PM), the function relating recall latency to displacement exhibits a symmetric $\mathrm{V}$-shaped function. When position marking is augmented with either response suppression (PM $+R S)$ or output interference $(\mathrm{PM}+\mathrm{OI})$, the function is rendered partially asymmetric, due to postponements having slightly shorter recall latencies than anticipations of the same absolute displacement. In sharp contrast to the above models, the combination of a primacy gradient with response suppression $(P G+R S)$ predicts a negative relationship between recall latency and displacement, with much 
faster recall latencies for postponements than for anticipations of the same absolute displacement. Finally, the model combining a primacy gradient, position marking, and response suppression (PG $+\mathrm{PM}+\mathrm{RS}$ ) also predicts a negative relationship between recall latency and displacement, but with a reduction in the slope of the function for postponements, compared to anticipations.

It can be seen by comparison of the model predictions in Figure 7 with the representative data shown in Figure 2 that the model comparisons confer support for a representational mechanism combining a primacy gradient, position marking, and response suppression.

3.4. Competitive queuing. The competitive queuing mechanism provides a powerful and parsimonious account of several key benchmark findings, most notably the greater incidence of transposition than item errors and the locality constraint underlying transpositions, as well as other ancillary outcomes, such as effects of primacy and recency of the serial position curve and the sequence length effect. All competitive queuing models predict that the most common errors will be movements and exchanges between items. This prediction is a natural consequence of the parallel sequence dynamics assumed by these models which when perturbed by noise will alter the relative priority of items. Near-neighbour transpositions predominate because the representation of serial order via an activation gradient necessarily implies that the strongest competitors to the target item at each recall position will be items from adjacent serial positions. These results are not unique to competitive queuing models-compound chaining models can produce the locality constraint on transpositions despite using a fundamentally serial —rather than parallel_-representation of serial order. However, electrophysiological recording studies with monkeys have provided strikingly direct evidence for the parallel sequence dynamics assumed by competitive queuing models (Averbeck, Chafee, Crowe, \& Georgopoulos, 2002; Averbeck, Chafee, Crowe, \& Georgopoulos, 2003; Averbeck, Crowe, Chafee, \& Georgopoulos, 2003). Furthermore, competitive queuing models have been successfully applied to a range of serial performance domains including action planning, music and speech production, saccade generation, motor sequence learning, spelling, and typing (see Hurlstone et al., 2014 for an extensive list of references). I therefore identify competitive queuing as the preferred mechanism for sequence planning and control in serial memory.

3.5. Response suppression. That response suppression contributes to serial recall is indicated by a number of indirect empirical precedents. First, the Ranschburg effect shows that people struggle to recall an item twice when it was repeated in a sequence (Crowder, 1968; Duncan \& Lewandowsky, 2005; Henson, 1998b; Jahnke, 1969; Vousden \& Brown, 1998), an outcome which according to response suppression accounts is attributable to the suppression of the repeated item once it is recalled, which renders it unlikely that it will be retrieved a second time. That this difficulty in recalling a repeated item twice is witnessed even when people can detect repetitions with a high level of accuracy (Henson, 1998b) suggests that response suppression is obligatory and not under volitional control. The operation of response suppression is further supported by the scarcity of erroneous repetitions in participants' serial reports (Henson, 1996; Hurlstone \& Hitch, 2015, 2018; Vousden \& Brown, 1998).

As well as incorporating response suppression to prevent perseveration on the same response during recall, many models rely on response suppression either partially (Brown et al., 2000; Burgess
\& Hitch, 1999; Hartley et al., 2016; Henson, 1998a; Lewandowsky \& Farrell, 2008a) or entirely (Farrell \& Lewandowsky, 2002; Grossberg \& Pearson, 2008; Page \& Norris, 1998) to produce recency. In those theories, response suppression contributes to recency by reducing the number of response competitors towards the end of the sequence, which increases the likelihood that the final item will be recalled in its correct position. A contribution of response suppression to recency in the forward recall of verbal sequences has been demonstrated in a conditional analysis of the recency effect by Farrell and Lewandowsky (2012). Across a large number of serial recall studies, these authors examined the accuracy of recall of the last item on those trials in which exactly two errors occurred in all but the final serial position. Specifically, they examined how the recency effect is modulated by three different combinations of errors, either: (1) two transpositions, (2) one transposition and one intrusion, or (3) two intrusions. In all instances, two errors are committed, but in (1) all items in the sequence have been recalled and hence suppressed, whereas in (2) the single intrusion leaves one item unrecalled and unsuppressed, whilst in (3) the two intrusions leave two items unrecalled and unsuppressed. Farrell and Lewandowsky found that the magnitude of the recency effect was a function of the number of items that have putatively been suppressed, with recency strongest following two transpositions, weaker following a transposition and an intrusion, and weakest following two intrusions. This outcome suggests that response suppression contributes to recency in verbal short-term memory.

However, it is important to note that studies that have dissociated the input and output positions of items in serial recall have highlighted the potential importance of factors other than response suppression as determinants of recency. For example, in a study by Cowan, Saults, Elliott, and Moreno (2002) participants were presented with 9-item verbal sequences and were post-cued to commence serial recall either at input position 1,4 , or 7 . When prompted to initiate recall from position 4 or 7 , recall proceeded up until the end of the sequence and then wrapped back around to the beginning. The latter two conditions permitted an analysis of serial position effects when the input and output order of items was dissociated. If recency is attributable to response suppression alone, then a recency effect should be witnessed over the output position, but not the input position, of items. However, Cowan and colleagues observed a strong recency effect over the input position of items, a result that can be attributed variously to (a) an "edge effect" (see earlier), (b) a positional coding mechanism in which the position markers for items near the end of the sequence are more distinctive than for items near the middle of the sequence (e.g.., Brown et al., 2007; Henson, 1998a), or (c) both factors. Taken together, the results of Farrell and Lewandowsky (2012) and Cowan et al. (2002) suggest that response suppression contributes to recency in serial recall but it is not the only contributing factor.

3.6. Output interference. A contribution of output interference to ordered recall would be reflected in the primacy effect. However, identifying such a contribution is rendered difficult by the fact that in the standard forward recall paradigm, the output order of items is perfectly correlated with their input order. Thus, the primacy effect may originate from input processes, such as a primacy gradient, output processes, such as output interference, or a combination of the two. As noted above, Cowan et al. (2002) empirically dissociated the input-output ordering of items by having participants commence serial recall from different input positions. In this study —and a kindred study by Oberauer (2003) that used a different paradigm to deconfound serial recall-a strong decrement 
in performance was observed over the output positions of items, suggesting that output interference is one source of the primacy effect in verbal short-term memory. However, it is noteworthy that in both studies a decrement in performance was also witnessed over the input positions of items, suggesting that a primacy gradient also contributes to the genesis of the primacy effect.

3.7. Similarity-sensitive encoding and retrieval. All accounts of item similarity effects have been designed to accommodate the classic phonological similarity effect in verbal serial recall. It follows that the different accounts cannot be distinguished on the basis of these data. However, adjudication becomes possible by considering their predictions concerning the effects of phonological similarity for sequences in which similar and dissimilar items are mixed together. The dissimilar immunity finding underlying the standard mixed-sequence similarity effect was initially taken as evidence in favour of retrieval-stage accounts of item similarity effects, since in those theories the recall of dissimilar items is unaffected by whether or not they are surrounded by similar items. However, the more recent demonstration of a mixed-sequence advantage for dissimilar items when guessing strategies are controlled is incompatible with theories that rely solely on the retrieval stage of recall for simulating the effects of item similarity. Qualified support for this claim was provided by Farrell and Lewandowsky (Farrell, 2006; Lewandowsky \& Farrell, 2008b) who showed by simulation that two such theories, namely SEM (Henson, 1998a) and the primacy model (Page \& Norris, 1998), were unable to account for the superior recall of dissimilar items on mixed sequences. However, this empirical pattern can be explained by the C-SOB model (Farrell, 2006; Lewandowsky \& Farrell, 2008a). The superior recall of dissimilar items on mixed sequences is predicted by C-SOB because its process of determining the encoding strength of each study item based on its similarity to existing information in memorysimilarity-sensitive encoding-means that similar items on mixed sequences will be encoded with less strength than their dissimilar counterparts on pure dissimilar sequences. Consequently, similar items on mixed sequences will be weaker competitors during recall than dissimilar items in corresponding positions on pure dissimilar sequences. The dissimilar items on mixed sequences therefore enjoy a recall advantage relative to their twins on pure sequences. Since C-SOB is the only existing model that provides a principled, quantitative account of the mixed-sequence advantage, I conclude that in verbal serial recall item similarity effects involve a combination of similarity-sensitive encoding and similarity-sensitive retrieval. Furthermore, because similarity-sensitive encoding also explains the genesis of a primacy gradient, I further conclude that the mixed-sequence advantage confers further support for the role of a primacy gradient in verbal serial recall.

However, it is important to emphasise that empirically the mixedsequence advantage for dissimilar items is small in magnitude, implying that the effect of item similarity at encoding is smaller than at retrieval. That the effect of similarity at encoding is small is buttressed by simulations conducted by Farrell (2006) comparing the quantitative fits of $\mathrm{C}-\mathrm{SOB}$ to the results of a mixed-sequence experiment using the standard implementation of the model in which similarity-sensitive encoding was operational and a control version in which it was switched off. The two versions of the model produced comparable phonological similarity effects for pure dissimilar and similar sequences (see Figures 8 and 9 of Farrell, 2006). This result suggests that even in C-SOB, similarity actually exerts its strongest effect during the retrieval stage, otherwise switching off similarity-sensitive encoding should have significantly reduced the size of the standard phonological similarity effect with pure dissimilar and similar sequences, compared to when it was operational. We can therefore conclude from these simulations that even in C-SOB the effect of similarity at encoding is comparatively small in relation to the effect at retrieval. On balance, therefore, the present evidence suggests that similarity affects both encoding and retrieval, but that by far the bigger effect is that on retrieval.

\section{Extending computational models of serial recall}

Before concluding this chapter, it merits comment that computational models of serial recall have been extended to account for a variety of phenomena, memory paradigms, and processes that are beyond the purview of the current chapter. For example, Burgess and Hitch (2006) and Page and Norris (2009) have presented augmented versions of their network model of the phonological loop and the primacy model, respectively, that can explain sequence learning, as witnessed in the Hebb repetition effect (Hebb, 1961) —-the long term learning of a sequence that is surreptitiously repeated every few trials. In these revised models, Hebb repetition learning is facilitated by a cumulative matching mechanism that incrementally matches an incoming sequence to previously presented sequences stored in memory.

Other theorists have examined whether mechanisms of serial recall considered in this chapter can serve as the foundation for explaining performance in kindred memory paradigms. On the one hand, Oberauer and colleagues (Oberauer, Lewandowsky, Farrell, Jarrold, \& Greaves, 2012; see also Oberauer \& Lewandowsky, 2011) have developed a computational model known as SOB-CSan extension of the C-SOB model of serial recall-that can explain performance in the complex span task of working memory in which the encoding of a sequence of items for serial recall is interspersed with a distracting processing task. The model inherits the core explanatory mechanisms of C-SOB-namely position marking, a primacy gradient, similarity-sensitive encoding, response suppression, and output interference. Additionally, it is assumed that distracting items from the processing task are encoded onto the position markers used to represent serial order, that this process is governed by the same similarity-sensitive encoding process that determines the encoding strength of to-be-remembered items, and that this interfering material can be partially removed using the same mechanism in the model that governs the implementation of response suppression.

On the other hand, based on the observation that in the free recall task-where participants can recall a sequence of items in any output order they choose-people exhibit a preference to recall information in forward serial order (Bhatarah, Ward, Smith, \& Hayes, 2009; Bhatarah, Ward, \& Tan, 2006, 2008; GrenfellEssam \& Ward, 2012; Ward, Tan, \& Grenfell-Essam, 2010), other theorists have attempted to model serial and free recall within a common explanatory framework (Brown et al., 2007; Farrell, 2012; Grossberg Pearson, 2008). For example, Farrell (2012) has shown that much serial and free recall phenomena can be explained in terms of a model embodying hierarchically organised position markers, a primacy gradient, response suppression, and output interference. A key assumption of the model is that for serial recall of short sequences (e.g., 6-items or less) items are encoded as a single group, whereas for free recall of longer sequences, items are spontaneously organised into multiple groups, with some variability in the number and size of groups. It is assumed that free recall of such longer sequences entails retrieving each group, one at a time, and recalling their contents in forward serial order. 
One exception is that the final group does not need to be actively retrieved and is instead automatically accessible at the start of the recall episode. These two assumptions are central to the model's ability to explain the contiguity and recency effects that have driven much theorising about free recall (see Chapter 5.5 of this handbook).

Finally, other theorists have used computational models of serial recall to evaluate whether forgetting in short-term memory is driven by temporal decay or interference, and to identify the role of rehearsal and/or attentional refreshing processes in shortterm memory and working memory paradigms (Lewandowsky \& Oberauer, 2015).

\section{Summary and conclusions}

- Serial recall is a deceptively simple task-the ease with which people accomplish it masks the wealth and complexity of findings this task has generated, and the computational theories that have been developed to account for them.

- I reviewed benchmark effects of serial recall observed across different content domains and interpreted them with reference to the mechanisms of serial recall instantiated in contemporary computational theories of serial memory.

- This analysis identified evidence for the operation of four common mechanisms of serial recall in the verbal, visual, and spatial domains-position marking, a primacy gradient, competitive queuing, and response suppression (Table 2).

- In verbal serial recall, there is evidence for both similaritysensitive encoding and retrieval, whereas in visual and spatial serial recall there is evidence for similarity-sensitive retrieval but appropriate tests of similarity-sensitive encoding are yet to be conducted.

- In verbal serial recall there is evidence that the retrieval of an item is accompanied by output interference, whereas the results that evince this mechanism are yet to be studied using visual or spatial serial recall.

- There is no direct evidence for the operation of associative chaining in verbal, visual, or spatial serial recall-indeed, the evidence in all three content domains questions the necessity of chaining.

\subsection{Open problems and future directions.}

- Some benchmarks listed in Table 1 (cf. Hurlstone et al., 2014, Table 1) have yet to be studied using visual or spatial serial recall. Where these benchmarks evince specific serial recall mechanisms, efforts should be made to determine whether these results generalise to the visual and spatial domains.

- Although associative chaining has been ruled out as a viable mechanism for short-term serial recall, there is some evidence for chaining in serial learning (Kahana, Mollison, \& Addis, 2010; Solway et al., 2012). Could it be that chaining begins to kick in during the long-term learning of sequences?

- Two major challenges for chaining accounts of serial recallrepeated items and mixed-sequence similarity effects-stem from the fact that chaining occurs between items. Would a model in which chaining operates between the different states of an evolving context signal-akin to the Temporal Context Model (Howard \& Kahana, 2002) of free recall (see Chapter 5.5 of this handbook) - fare better with respect to the data?
- How do the different mechanisms of serial recall evinced by the current analysis map onto the four components of the working memory model (Baddeley, 2000, 2007)?

- Hartley et al. (2016) suggest the context-timing signal in the BUMP model provides a stepping stone toward a general theory of serial order in language processing, potentially linking speech perception, speech production, and verbal short-term memory through their common dependence on rhythm and timing. To what extent can the BUMP mechanism explain phenomena of serial order in language more generally, such as the segmentation of speech, and errors in speech production?

- Positional models of serial recall assume a central serial ordering mechanism not tied to any specific input modality. The BUMP mechanism (Hartley et al., 2016) can be seen as as a front-end to such models that enables them to deal with spoken inputs. This in turn highlights the importance of specifying corresponding front-ends for other modalities (e.g., visual, spatial).

\section{References}

Aaronson, D. (1968). Temporal course of perception in an immediate recall task. Journal of Experimental Psychology, 76, 129-140.

Agam, Y., Bullock, D., \& Sekuler, R. (2005). Imitating unfamiliar sequences of connected linear motions. Journal of Neurophysiology, 94, 2832-2843.

Agam, Y., Galperin, H., Gold, B. J., \& Sekuler, R. (2007). Learning to imitate novel motion sequences. Journal of Vision, 7 , 1-17.

Agam, Y., Huang, J., \& Sekuler, R. (2010). Neural correlates of sequence encoding in visuomotor learning. Journal of Neurophysiology, 103, 1418-1424.

Anderson, J. R., Bothell, D., Lebiere, C., \& Matessa, M. (1998). An integrated theory of list memory. Journal of Memory and Language, 38, 341-380. doi:10.1006/jmla.1997.2553.

Averbeck, B. B., Chafee, M. V., Crowe, D. A., \& Georgopoulos, A. P. (2002). Parallel processing of serial movements in prefrontal cortex. Proceedings of the National Academy of Sciences, 99, $13172-13177$.

Averbeck, B. B., Chafee, M. V., Crowe, D. A., \& Georgopoulos, A. P. (2003). Neural activity in prefrontal cortex during copying geometrical shapes I: Single cells encode shape, sequence, and metric parameters. Experimental Brain Research, 150, 127-141.

Averbeck, B. B., Crowe, D. A., Chafee, M. V., \& Georgopoulos, A. P. (2003). Neural activity in prefrontal cortex during copying geometrical shapes II: Decoding shape segments from neural ensembles. Experimental Brain Research, 150, 142-153.

Avons, S. E. (1998). Serial report and item recognition of novel visual patterns. British Journal of Psychology, 89, 285-308.

Avons, S. E. (2007). Spatial span under translation: A study of reference frames. Memory and Cognition, 35, 402-417.

Avons, S. E. \& Mason, A. (1999). Effects of visual similarity on serial report and item recognition. Quarterly Journal of Experimental Psychology Section A, 52, 217-240.

Baddeley, A. D. (1968). How does acoustic similarity influence short-term memory? Quarterly Journal of Experimental Psychology, 20, 249-263.

Baddeley, A. D. (1986). Working Memory. Oxford: Clarendon Press. 
Baddeley, A. D. (2000). The episodic buffer: A new component of working memory? Trends in Cognitive Sciences, 4, 417-423.

Baddeley, A. D. (2007). Working memory, thought and action. Oxford: Oxford University Press.

Baddeley, A. D., \& Hitch, G. J. (1974). Working memory. In G. A. Bower (Ed.), Recent advances in learning and motivation (Vol. 8, pp. 47-89). New York: Academic Press.

Baddeley, A. D., Gathercole, S. E., \& Papagno, C. (1998). The phonological loop as a language learning device. Psychological Review, 105, 158-173.

Bhatarah, P., Ward, G., Smith, J., \& Hayes, L. (2009). Examining the relationship between free recall and immediate serial recall: Similar patterns of rehearsal and similar effects of word length, presentation rate, and articulatory suppression. Memory \& Cognition, 37, 689-713.

Bhatarah, P., Ward, G., \& Tan, L. (2006). Examining the relationship between free recall and immediate serial recall: The effect of concurrent task performance. Journal of Experimental Psychology: Learning, Memory, and Cognition, 32, 215-229.

Bhatarah, P., Ward, G., \& Tan, L. (2008). Examining the relationship between free recall and immediate serial recall: The serial nature of recall and the effect of test expectancy. Memory \& Cognition, 36, 20-34.

Botvinick, M. M., \& Plaut, D. C. (2006). Short-term memory for serial order: A recurrent neural network model. Psychological Review, 113, 201-233.

Brown, G. D. A., Neath, I., \& Chater, N. (2007). A temporal ratio model of memory. Psychological Review, 114, 539-576.

Brown, G. D. A., Preece, T., \& Hulme, C. (2000). Oscillator-based memory for serial order. Psychological Review, 107, 127181.

Burgess, N., \& Hitch, G. J. (1992). Towards a network model of the articulatory loop. Journal of Memory and Language, 31, 429-460.

Burgess, N., \& Hitch, G. (1999). Memory for serial order: A network model of the phonological loop and its timing. Psychological Review, 106, 551-581.

Burgess, N., \& Hitch, G. J. (2006). A revised model of shortterm memory and long-term learning of verbal sequences. Journal of Memory and Language 55, 627-652.

Conrad, R. (1960). Serial order intrusions in immediate memory. British Journal of Psychology, 50, 349-359.

Conrad, R. (1964). Acoustic confusions in immediate memory. British Journal of Psychology, 55, 75-84.

Cowan, N., Saults, S., Elliott, E. M., \& Moreno, M. (2002). Deconfounding serial recall. Journal of Memory and Language, 46, 153-177.

Crannell, C. W., \& Parrish, J. N. (1957). A comparison of immediate memory span for digits, letters, and words. Journal of Psychology, 44, 319-327.

Crowder, R. G. (1968). Intraserial repetition effects in immediate memory. Journal of Verbal Learning and Verbal Behavior, 7, 446-451.

Dell, G. S., Burger, L. K., \& Svec, W. R. (1997). Language production and serial order: A functional analysis and a model. Psychological Review, 104, 123-147. doi:10.1037//0033295X.104.1.123

Duncan, M., \& Lewandowsky, S. (2005). The time course of response suppression: No evidence for a gradual release from inhibition. Memory, 13, 236-246.
Ebbinghaus, H. (1885/1964). Memory: A contribution to experimental psychology. New York: Dover.

Estes, W. K. (1972). An associative basis for coding and organization in memory. In A. W. Melton \& E. Martin (Eds.), Coding processes in human memory (pp. 161-190). Washington, DC: V.H. Winston \& Sons.

Farrand, P., Parmentier, F. B. R., \& Jones, D. M. (2001). Temporalspatial memory: Retrieval of spatial information does not reduce recency. Acta Psychologica, 106, 285-301.

Farrell, S. (2006). Mixed-list phonological similarity effects in delayed serial recall. Journal of Memory and Language, 55, 587-600.

Farrell, S. (2008). Multiple roles for time in short-term memory: Evidence from serial recall of order and timing. Journal of Experimental Psychology: Learning, Memory, and Cognition, 34, 128-145.

Farrell, S. (2012). Temporal clustering and sequencing in shortterm memory and episodic memory. Psychological Review, 119, 223-271.

Farrell, S., Hurlstone, M. J., \& Lewandowsky, S. (2013). Sequential dependencies in recall of sequences: Filling in the blanks. Memory \& Cognition.

Farrell, S., \& Lewandowsky, S. (2002). An endogenous distributed model of ordering in serial recall. Psychonomic Bulletin \& Review, 9, 59-79.

Farrell, S., \& Lewandowsky, S. (2003). Dissimilar items benefit from phonological similarity in serial recall. Journal of Experimental Psychology: Learning, Memory, and Cognition, 29, 838-849.

Farrell, S., \& Lewandowsky, S. (2004). Modelling transposition latencies: Constraints for theories of serial order memory. Journal of Memory and Language, 51, 115-135.

Farrell, S., \& Lewandowsky, S. (2012). Response suppression contributes to recency in serial recall. Memory \& Cognition, 40, 1070-1080.

Farrell, S., Wise, V., \& Lelièvre, A. (2011). Relations between timing, position, and grouping in short-term memory. Memory \& Cognition, 39, 573-587.

Frankish, C. (1985). Modality-specific grouping effects in shortterm memory. Journal of Memory and Language, 24, 200209.

Frankish, C. (1989). Perceptual organization and precategorical acoustic storage. Journal of Experimental Psychology: Learning, Memory, and Cognition, 15, 469-479.

Gathercole, S. E., \& Baddeley, A. D. (1990). The role of phonological memory in vocabulary acquisition: A study of young children learning new names. British Journal of Psychology, 81, 439-454.

Glasspool, D. W. (2005). Modelling serial order in behaviour: Evidence from performance slips. In G. Houghton (Ed.), Connectionist models in cognitive psychology (pp. 241-270). Hove: Psychology Press.

Grenfell-Essam, R., \& Ward, G. (2012). Examining the relationship between free recall and immediate serial recall: The role of list length, strategy use, and test expectancy. Journal of Memory and Language, 67, 106-148.

Groeger, J. A., Banks, A. P., \& Simpson, P. J. (2008). Serial memory for sound-specified locations: Effects of spatial uncertainty and motor suppression. Quarterly Journal of Experimental Psychology, 61, 248-262. 
Grossberg, S. (1978a). A theory of human memory: Selforganization and performance of sensory-motor codes, maps, and plans. In R. Rosen \& Snell (Eds.), Progress in theoretical biology (Vol. 5, pp. 233-374). New York: Academic Press.

Grossberg, S. (1978b). Behavioral contrast in short-term memory: Serial binary memory models or parallel continuous memory models? Journal of Mathematical Psychology, 17, 199-219.

Grossberg, S., \& Pearson, L. R. (2008). Laminar cortical dynamics of cognitive and motor working memory, sequence learning and performance: Towards a unified theory of how the cerebral cortex works. Psychological Review, 115, 677-732.

Guérard, K., \& Tremblay, S. (2008). Revisiting evidence for functional equivalence across verbal and spatial domains in memory. Journal of Experimental Psychology: Learning, Memory, and Cognition, 34, 556-569.

Hartley, T., Hurlstone, M. J., \& Hitch, G. J. (2016). Effects of rhythm on memory for spoken sequences: A model and tests of its stimulus-driven mechanism. Cognitive Psychology, 87, 135178.

Hebb, D. O. (1961). Distinctive features of learning in the higher animal. In J. F. Delafresnaye (Ed.), Brain mechanisms and learning (pp. 37-46). New York, NY: Oxford University Press.

Healy, A. F. (1982). Short-term memory for order information. In G. $\mathrm{H}$. Bower (Ed.), The psychology of learning and motivation (Vol. 16, pp. 191-238). New York: Academic Press.

Healy, A. F., Fendrich, D. W., Cunningham, T. F., \& Till, R. E. (1987). Effects of cuing on short-term retention of order information. Journal of Experimental Psychology: Learning, Memory, and Cognition, 13, 413-425.

Henson, R. N. A. (1996). Short-term memory for serial order. Unpublished doctoral thesis. Cambridge University, Cambridge, U.K.

Henson R. N. A. (1998a). Short-term memory for serial order: The start-end model. Cognitive Psychology, 36, 73-137.

Henson, R. N. A. (1998b). Item repetition in short-term memory: Ranschburg repeated. Journal of Experimental Psychology: Learning, Memory, and Cognition, 24, 1162-1181.

Henson, R. N. A. (1999). Positional information in short-term memory: Relative or absolute? Memory \& Cognition, 27, 915-927.

Henson, R. N., Norris, D. G., Page, M. P. A., \& Baddeley, A. D. (1996). Unchained memory: Error patterns rule out chaining models of immediate serial recall. Quarterly Journal of Experimental Psychology, 49A, 80-115.

Hitch, G. J., Burgess, N., Towse, J. N., \& Culpin, V. (1996). Temporal grouping effects in immediate recall: A working memory analysis. Quarterly Journal of Experimental Psychology, 49, 116-139.

Houghton, G. (1990). The problem of serial order: A neural network model of sequence learning and recall. In R. Dale, C. Mellish, \& M. Zock, (Eds.), Current Research in Natural Language Generation (pp. 287-319). London: Academic Press.

Howard, M. W., \& Kahana, M. J. (2002). A distributed representation of temporal context. Journal of Mathematical Psychology, 46, 269-299.

Hurlstone, M. J., (2018). Functional similarities and differences between the coding of positional information in verbal and spatial short-term memory. Memory, 14, 1-16.

Hurlstone, M. J., \& Hitch, G. J. (2015). How is the serial order of a spatial sequence represented? Insights from transposition latencies. Journal of Experimental Psychology: Learning, Memory, and Cognition, 41, 295-324.

Hurlstone, M. J., \& Hitch, G. J. (2018). How is the serial order of a visual sequence represented? Insights from transposition latencies. Journal of Experimental Psychology: Learning, Memory, and Cognition, 44(2), 167-192.

Hurlstone, M. J., Hitch, G. J., \& Baddeley, A. D. (2014). Memory for serial order across domains: An overview of the literature and directions for future research. Psychological Bulletin, 140, 339-373.

Jahnke, J. C. (1969). The Ranschburg effect. Psychological Review, 76, 592-605.

Jalbert, A., Saint-Aubin, J., \& Tremblay, S. (2008). Visual similarity in short-term recall for where and when. Quarterly Journal of Experimental Psychology, 61, 353-360.

Jones, D. M., Farrand, P., Stuart, G., \& Morris, N. (1995). Functional equivalence of verbal and spatial information in serial short-term memory. Journal of Experimental Psychology: Learning, Memory, and Cognition, 21, 1008-1018.

Kahana, M. J. (2012). Foundations of human memory. New York: Oxford University Press.

Kahana, M. J. \& Jacobs, J. (2000) Interresponse times in serial recall: Effects of intraserial repetition. Journal of Experimental Psychology: Learning, Memory and Cognition, 26, 1188-1197.

Kahana, M. J., Mollison, M. V., \& Addis, K. M. (2010). Positional cues in serial learning: The spin-list technique. Memory \& Cognition, 38(1), 92-101.

Lashley, K. (1951). The problem of serial order in behavior. In L. A. Jeffress (Ed.), Cerebral mechanisms in behavior (pp.112136). New York: Wiley.

Leclercq, A., \& Majerus, S. (2010). Serial-order short-term memory predicts vocabulary development: Evidence from a longitudinal study. Developmental Psychology, 46, 417-427.

Lewandowsky, S., \& Brown, G. D. A. (2005). Serial recall and presentation schedule: a micro-analysis of local distinctiveness. Memory, 13, 283-292.

Lewandowsky, S., Brown, G. D. A., Wright, T., Nimmo, L. M. (2006). Timeless memory: evidence against temporal distinctiveness models of short-term memory for serial order. Journal of Memory and Language, 54, 20-38.

Lewandowsky, S., \& Farrell, S. (2008a). Short-term memory: New data and a model. The Psychology of Learning and Motivation, 49, 1-48.

Lewandowsky, S., \& Farrell, S. (2008b). Phonological similarity in serial recall: Constraints on theories of memory. Journal of Memory and Language, 58, 429-448.

Lewandowsky, S., \& Murdock, B. B. (1989). Memory for serial order. Psychological Review, 96, 25-57.

Lewandowsky, S., \& Oberauer, K. (2015). Rehearsal in serial recall: An unworkable solution to the nonexistent problem of decay. Psychological Review, 122(4), 674-699.

Logan, G. (2018). Automatic control: How experts act without thinking. Psychological Review, 125(4), 453-485.

Logie, R. (1995). Visuo-spatial working memory. Hove: Lawrence Erlbaum Associates.

Logie, R. H., Saito, S., Morita, A., Varma, S., \& Norris, D. (2016). Recalling visual serial order for verbal sequences. Memory \& Cognition, 44, 590-607. 
Majerus, S., Poncelet, M., Elsen, B., \& Van der Linden, M. (2006). Exploring the relationship between verbal short-term memory for serial order and item information and new word learning in adults. European Journal of Cognitive Psychology, 18, 848-873.

Maybery, M., Parmentier, F. B. R., \& Jones, D. M. (2002). Grouping of list items reflected in the timing of recall: Implications for models of serial verbal memory. Journal of Memory and Language, 47, 360-385.

Meulenbroek, R. G. J., Thomassen, A. J. W. M., Schillings, J. J., \& Rosenbaum, D. A. (1996). Synergies and sequencing in copying L-shaped patterns. Handwriting and drawing research: Basic and applied issues, 41-55.

Murdock, B. B. (1993). TODAM2: A model for the storage and retrieval of item, associative, and serial-order information. Psychological Review, 100, 183-203.

Murdock, B. B. (1995). Developing TODAM: Three models for serial order information. Memory \& Cognition, 23, 631-645.

Neath, I., \& Crowder, R. G. (1990). Schedules of presentation and temporal distinctiveness in human memory. Journal of Experimental Psychology: Learning, Memory, and Cognition, 16(2), 316-327.

Neath, I., \& Crowder, R. G. (1996). Distinctiveness and very short-term serial position effects. Memory, 4, 225-242.

Ng, L. H., \& Maybery, M. T. (2002). Grouping in verbal short-term memory: Is position coded temporally? Quarterly Journal of Experimental Psychology: Human Experimental Psychology, 55A, 391-424.

Ng, L. H., \& Maybery, M. T. (2005). Grouping in short-term memory: Do oscillators code the positions of items? Journal of Experimental Psychology: Learning, Memory, and Cognition, 31, 175-181.

Nimmo, L. M., \& Lewandowsky, S. (2005). From brief gaps to very long pauses: temporal isolation does not benefit serial recall. Psychonomic Bulletin \& Review, 12, 999-1004.

Nimmo, L. M., \& Lewandowsky, S. (2006). Distinctiveness revisited: unpredictable temporal isolation does not benefit 1208 short-term serial recall of heard or seen events. Memory \& Cognition, 34, 1368-1375.

Oberauer, K. (2003). Understanding serial position curves in short-term recognition and recall. Journal of Memory and Language, 49, 469-483.

Oberauer, K., \& Lewandowsky, S. (2011). Modeling working memory: A computational implementation of the time-based resource sharing theory. Psychonomic Bulletin \& Review, 18, 10-45.

Oberauer, K., Lewandowsky, S., Awh, E., Brown, G. D., Conway, A., Cowan, N., ... \& Ma, W. J. (2018). Benchmarks for models of short-term and working memory. Psychological Bulletin, 144(9), 885-958.

Oberauer, K., Lewandowsky, S., Farrell, S., Jarrold, C., \& Greaves, M. (2012). Modeling working memory: An interference model of complex span. Psychonomic Bulletin \& Review, 19, 779819.

Osth, A. F., \& Dennis, S. (2015a). The fill-in effect in serial recall can be obscured by omission errors. Journal of Experimental Psychology: Learning, Memory, and Cognition, 41(5), 14471455.

Osth, A. F., \& Dennis, S. (2015b). Prior-list intrusions in serial recall are positional. Journal of Experimental Psychology: Learning, Memory, and Cognition, 41(6), 1893-1901.
Page, M. P. A., \& Norris, D. (1998). The primacy model: A new model of immediate serial recall. Psychological Review, 105, 761-781.

Page, M. P. A., \& Norris, D. (2009). A model linking immediate serial recall, the Hebb repetition effect and the learning of phonological word forms. Philosophical Transactions of The Royal Society B, 364, 3737-3753.

Palmer, C., \& Pfordresher, P. Q. (2003). Incremental planning in sequence production. Psychological Review, 110, 683-712.

Parmentier, F. B. R., Andrés, P., Elford, G., \& Jones, D. M. (2006). Organization of visuo-spatial serial memory: Interaction of temporal order with spatial and temporal grouping. Psychological Research, 70, 200-217.

Parmentier, F. B. R., \& Jones, D. M. (2000). Functional characteristics of auditory temporal-spatial memory: Evidence from serial order errors. Journal of Experimental Psychology: Learning, Memory, and Cognition, 26, 222-238.

Parmentier, F. B. R., King, S., \& Dennis, I. (2006). Local temporal distinctiveness does not benefit auditory and spatial serial recall. Psychonomic Bulletin \& Review, 13, 458-465.

Parmentier, F. B. R., Maybery, M., \& Jones, D. M. (2004). Temporal grouping in auditory spatial serial memory. Psychonomic Bulletin \& Review, 11, 501-507.

Peteranderl, S., \& Oberauer, K. (2018). Serial recall of colors: Two models of memory for serial order applied to continuous visual stimuli. Memory \& Cognition, 46(1), 1-16.

Ryan, J. (1969a). Grouping and short-term memory: Different means and patterns of grouping. Quarterly Journal of Experimental Psychology, 21, 137-147.

Ryan, J. (1969b). Temporal grouping, rehearsal and short-term memory. Quarterly Journal of Experimental Psychology, 21, 148-155.

Smyth, M. M. (1996). Interference with rehearsal in spatial working memory in the absence of eye movements. Quarterly Journal of Experimental Psychology, 40A, 940-949.

Smyth, M. M., Hay, D. C., Hitch, G. J., \& Horton, N. J. (2005). Serial position memory in the visual-spatial domain: Reconstructing sequences of unfamiliar faces. Quarterly Journal of Experimental Psychology, 58, 909-930.

Smyth, M. M., Pearson N. A., \& Pendleton, L. R. (1988). Movement and working memory: Patterns and positions in space. Quarterly Journal of Experimental Psychology, 40A, 497514.

Smyth, M. M., \& Scholey, K. A. (1994). Interference in spatial immediate memory. Memory \& Cognition, 22, 1-13.

Smyth, M. M., \& Scholey, K. A. (1996). Serial order in spatial immediate memory. Quarterly Journal of Experimental Psychology, 49, 159-177.

Solway, A., Murdock, B. B., \& Kahana, M. J. (2012). Positional and temporal clustering in serial order memory. Memory \& Cognition, 40, 177-190.

Surprenant, A. M., Kelley, M. R., Farley, L. A., \& Neath, I. (2005). Fill-in and infill errors in order memory. Memory, 13, 267-273.

Tremblay, S., Guérard, K., Parmentier, F. B. R., Nicholls, A. P., \& Jones, D. M. (2006). A spatial modality effect in serial memory. Journal of Experimental Psychology: Learning, Memory, and Cognition, 32, 1208-1215.

Vousden, J. I., \& Brown, G. D. A. (1998). To repeat or not to repeat: The time course of response suppression in sequential behaviour. In J. A. Bullinaria, D. W. Glasspool, \& G. Houghton (Eds.), Proceedings of the fourth neural computation and 
psychology workshop: Connectionist representations (pp. 301-315). London: Springer Verlag.

Ward, G., Avons, S. E., \& Melling, L. (2005). Serial position curves in short-term memory: Functional equivalence across modalities. Memory, 13, 308-317.

Ward, G., Tan, L., \& Grenfell-Essam, R. (2010). Examining the relationship between free recall and immediate serial recall: The effect of list length and output order. Journal of Experimental Psychology: Learning, Memory, and Cognition, 36, 1207-1241

Wickelgren, W. A. (1965a). Acoustic similarity and retroactive interference in short-term memory. Journal of Verbal Learning and Verbal Behavior, 4, 53-61.

Wickelgren, W. A. (1965b). Short-term memory for phonemically similar lists. American Journal of Psychology, 78, 567-574. 